\title{
THE INTRINSIC SHAPE OF SPIRAL GALAXIES IN THE 2MASS LARGE GALAXY ATLAS
}

\author{
BARBARA S. RYDEN \\ Department of Astronomy, Ohio State University, 140 West 18th Avenue, Columbus, OH 43210; ryden@astronomy.ohio-state.edu \\ Received 2005 August 5; accepted 2005 December 14
}

\begin{abstract}
The apparent shapes of spiral galaxies in the Two Micron All Sky Survey Large Galaxy Atlas are used to constrain the intrinsic shapes of their disks. When the distribution of apparent axis ratios is estimated using a nonparametric kernel method, the shape distribution is inconsistent with axisymmetry at the $90 \%$ confidence level in the $B$ band and at the $99 \%$ confidence level in the $K_{s}$ band. If spirals are subdivided by Hubble type, the late-type spirals (Sc and later) are consistent with axisymmetry, while the earlier spirals are strongly inconsistent with axisymmetry. The distribution of disk ellipticity can be fitted adequately with either a Gaussian or a lognormal distribution. The best fits for the late spirals imply a median ellipticity of $\epsilon \approx 0.07$ in the $B$ band and $\epsilon \approx 0.02$ in the $K_{s}$ band. For the earlier spirals, the best fits imply a median ellipticity of $\epsilon \approx 0.18$ in the $B$ band and $\epsilon \approx 0.30$ in the $K_{s}$ band. The observed scatter in the Tully-Fisher relation, for both late and early spirals, is consistent with the disk ellipticity measured in the $B$ band. This indicates that excluding spirals of Hubble type earlier than Sc will minimize the intrinsic scatter in the Tully-Fisher relation used as a distance indicator.
\end{abstract}

Subject headings: galaxies: fundamental parameters — galaxies: photometry — galaxies: spiral

\section{INTRODUCTION}

The intrinsic shapes of disks in spiral galaxies, although difficult to determine, are useful to know. In particular, the ellipticity of a disk in its outer region reflects the nonaxisymmetry of the potential in which it exists and thus provides information about the shape of dark matter halos. Noncircular disks produce scatter in the Tully-Fisher relation between rotation velocity and absolute magnitude (Tully \& Fisher 1977; Franx \& de Zeeuw 1992); therefore, knowledge of the disk ellipticity helps in understanding the origin of scatter in the Tully-Fisher relation. In particular, the usefulness of the Tully-Fisher relation as a distance indicator would be enhanced for a population of disk galaxies with small intrinsic ellipticity. If the shape of a disk is approximated as an ellipsoid with principal axes of length $a \geq b \geq c$, the disk shape can be described by two parameters: the dimensionless disk thickness, $\gamma \equiv c / a$, and the disk ellipticity, $\epsilon \equiv 1-b / a$. The simplest approximation, that disks are infinitesimally thin and perfectly circular $(\gamma=0, \epsilon=0)$, is occasionally useful; for many purposes, however, it is useful to have a more accurate estimate of the shapes of disks.

The thickness of disks can be determined from catalogs of edge-on spiral galaxies, such as the Flat Galaxy Catalogue (Karachentsev et al. 1993) and the Revised Flat Galaxy Catalogue (Karachentsev et al. 1999). Some superthin galaxies have axis ratios as small as $\gamma \approx 0.05$ (Goad \& Roberts 1981). The study of Guthrie (1992), using images of edge-on spirals from blue Palomar Sky Survey plates, found that $\log q$ had a mean value of -0.95 . The mean disk thickness depends on the spirals' morphological type (Bottinelli et al. 1983); Guthrie (1992) found that the mean value of $\log q$ ranges from -0.7 for edge-on $\mathrm{Sa}$ galaxies to -1.1 for edge-on Sd galaxies. The disk thickness of an individual spiral galaxy also depends on the wavelength at which it is observed. Edge-on spirals have strong vertical color gradients, becoming much redder with increasing height above the midplane (Dalcanton \& Bernstein 2002). This gradient reflects the transition from the thin disk, with its young blue stellar population, to the thick disk, with its older redder population. As a consequence, $q$ of an edge-on galaxy becomes larger at longer wavelengths. For example, the galaxies in the Dalcanton $\&$ Bernstein (2000) sample were chosen to have $q \leq 0.125$ at blue wavelengths; in deep $R$-band images, the typical axis ratio increases to $q \approx 0.23$ (Dalcanton \& Bernstein 2002). Similarly, Mitronova et al. (2004) found that edge-on spirals that have $q \approx 0.17$ in blue images have $q \approx 0.35$ in $2 \mu$ m images.

Estimates of the distribution of disk ellipticity $\epsilon$ can be made from the distribution of apparent axis ratios $q$ for a large population of randomly oriented spiral galaxies. The signature of nonzero ellipticity, in this case, is a scarcity of nearly circular $(q \approx 1)$ spiral galaxies. Binney \& de Vaucouleurs (1981), using spiral galaxies from the Second Reference Catalogue of Bright Galaxies (de Vaucouleurs et al. 1976), concluded that late-type spirals were better fitted by slightly elliptical disks $(\epsilon \approx 0.1)$ than by perfectly circular disks. More recent studies (Grosbøl 1985; Lambas et al. 1992; Fasano et al. 1993; Alam \& Ryden 2002; Ryden 2004) have confirmed the ellipticity of disks and have permitted estimates of the distribution function for intrinsic ellipticity. The distribution of $\epsilon$ is commonly fitted with a halfGaussian peaking at $\epsilon=0$. Values of $\sigma_{\epsilon}$ in this case range from $\sigma_{\epsilon}=0.12$ (Lambas et al. 1992) through $\sigma_{\epsilon}=0.13$ (Fasano et al. 1993) to $\sigma_{\epsilon}=0.21$ (Ryden 2004).

Kinematic studies can also provide an estimate of the ellipticity of the gravitational potential in the disk plane. In an elliptical potential with small ellipticity $\epsilon_{\Phi}$, the closed stellar orbits will themselves be ellipses (Binney 1978). When the resulting elliptical stellar disk is seen in projection, the isophotal principal axes will be misaligned with the kinematic principal axes. Because of the misalignment, there will be a velocity gradient along the isophotal minor axis proportional to $\epsilon_{\Phi} \sin 2 \phi$, where $\phi$ is the azimuthal viewing angle relative to the long axis of the potential (Franx \& de Zeeuw 1992). Measurement of the kinematic misalignment for a sample of nine disk galaxies yielded an average ellipticity of $\epsilon_{\Phi} \approx 0.044$ (Schoenmakers et al. 1997; Schoenmakers 1998). A similar analysis of gas kinematics in seven disk galaxies by Beauvais \& Bothun (1999) found an average ellipticity of $\epsilon \approx 0.059$ for the gas orbits. Andersen \& Bershady (2002), using the method of Andersen et al. (2001), combined twodimensional kinematic and photometric data for a sample of 
28 nearly face-on disk galaxies; they found an average halo ellipticity of $\epsilon_{\Phi} \approx 0.054$ and an average disk ellipticity of $\epsilon \approx 0.076$ for their sample. Taking into account the selection criteria of Andersen \& Bershady (2002), the implied median disk ellipticity is $\epsilon \approx 0.10$ (Ryden 2004).

Much of what we know about the ellipticity of disks thus comes from looking at galaxies with low inclination; much of what we know about disk thickness comes from looking at galaxies with high inclination. In principle, both ellipticity and thickness can be determined by looking at a sample of disk galaxies with random inclinations (Fasano et al. 1993). However, at visible wavelengths, thanks to the effects of dust, it is difficult to create a sample in which the disk inclinations are completely random. Because disk galaxies are not fully transparent, a flux-limited sample will be deficient in nearly edge-on galaxies (Huizinga \& van Albada 1992). Because disk galaxies are not fully opaque, an angular-diameter-limited sample will have an excess of nearly edge-on galaxies (Huizinga \& van Albada 1992; Masters et al. 2003).

To minimize the effects of dust, I examine the apparent shapes of disk galaxies in the Two Micron All-Sky Survey (2MASS). In $\S 2$, I extract a randomly inclined sample of spiral galaxies from the 2MASS Large Galaxy Atlas (LGA). In $\S 3$, I make both nonparametric and parametric estimates of $f(\gamma)$, the distribution of disk thickness, assuming the disks are axisymmetric. Separate estimates are made for early-type spirals and for late-type spirals. In $\S 4$, I make nonparametric estimates of $f(\epsilon)$, the distribution of disk ellipticity for nonaxisymmetric disks. Both Gaussian and lognormal functions are found to give satisfactory fits to $f(\epsilon)$. In $\S 5$, I examine the influence of the deduced disk ellipticity on the scatter of the Tully-Fisher relation and consider the possible origins of disk ellipticity for early-type and late-type spirals.

\section{THE GALAXY SAMPLE}

The 2MASS Large Galaxy Atlas (LGA) is a catalog of galaxies with large angular size as seen in the near-infrared (Jarrett et al. 2003). The LGA provides an essentially complete sample of galaxies with $R_{20} \geq 60^{\prime \prime}$, where $R_{20}$ is the semimajor axis of the $K_{s}$ band $20 \mathrm{mag} \operatorname{arcsec}^{-2}$ isophote. Observations in the $K_{s}$ band, with an effective wavelength of $2.2 \mu \mathrm{m}$, have two advantages for the study of galaxy morphology. First, the effects of dust are minimized. Second, the light in the $K_{s}$ band emphasizes the smoother distribution of Population II stars rather than the patchier distribution of Population I stars, which tend to lie along spiral arms. The LGA galaxy images consist of individual 2MASS coadded images mosaicked together. The typical $1 \sigma$ background noise in a single $K_{s}$ co-added image is $\sim 20 \mathrm{mag} \mathrm{arcsec}{ }^{-2}$; the point-spread function FWHM varies from $2^{\prime \prime}$ to $3^{\prime \prime}$, depending on seeing (Jarrett et al. 2003). The online Large Galaxy Atlas, ${ }^{1}$ version 2.0, contains 620 galaxies. To create a sample of spiral galaxies, I selected those galaxies with morphological classification SA, SAB, SB, or S. Galaxies labeled "pec" were excluded, as were $\mathrm{S} 0$ galaxies. The resulting sample contains 383 spiral galaxies.

The apparent shape of each galaxy in the $K_{s}$ band is estimated by Jarrett et al. (2003) from the axis ratio of the $3 \sigma$ intensity isophote, corresponding to a surface brightness of $\mu_{K} \approx 18.8 \mathrm{mag} \mathrm{arcsec}^{-2}$; I call this axis ratio $q_{K}$. For purposes of comparison, the apparent axis ratio $q_{B}$ in the $B$ band was estimated by taking the axis ratio of the $\mu_{B}=25$ mag $\operatorname{arcsec}^{-2}$ isophote from the LEDA database. Be-

\footnotetext{
${ }^{1}$ See http://irsa.ipac.caltech.edu/applications/2MASS/LGA.
}

cause the 2MASS LGA is an angular diameter-limited sample and spiral galaxies are largely transparent in the $K_{s}$ band, the LGA contains an excess of high-inclination disks with small $q_{K}$. For instance, of the 30 LGA galaxies with the largest value of $R_{20}$, 10 are spiral galaxies with $q_{K}<0.33$.

To account for inclination effects, we must estimate what the isophotal radius of each galaxy would be if we saw it face-on. Suppose, to begin, that the isoluminosity surfaces of the galaxy are adequately approximated as similar concentric coaxial ellipsoids, given by the formula

$$
m^{2}=x^{2}+y^{2} / \beta^{2}+z^{2} / \gamma^{2}
$$

where $1 \geq \beta \geq \gamma$. The galaxy is viewed from an angle $(\theta, \phi)$, where $\theta$ is the polar angle measured from the positive $z$-axis and $\phi$ is the azimuthal angle measured from the positive $x$-axis. Assuming perfect transparency, the isophotes of the galaxy will be ellipses, given by the formula

$$
M^{2}=D X^{2}+E Y^{2} .
$$

If I define, following Binney (1978),

$$
\begin{gathered}
A(\theta, \phi)=\cos ^{2} \theta\left(\beta^{2} \sin ^{2} \phi+\cos ^{2} \phi\right)+\gamma^{2} \sin ^{2} \theta, \\
B(\theta, \phi)=\left(1-\beta^{2}\right) \cos \theta \sin 2 \phi \\
C(\phi)=\sin ^{2} \phi+\beta^{2} \cos ^{2} \phi, \\
f(\theta, \phi)=\gamma^{2} \sin ^{2} \theta\left(\beta^{2} \cos ^{2} \phi+\sin ^{2} \phi\right)+\beta^{2} \cos ^{2} \theta,
\end{gathered}
$$

then

$$
\begin{aligned}
& D(\theta, \phi)=\frac{1}{2 f}\left[A+C-\sqrt{(A-C)^{2}+B^{2}}\right], \\
& E(\theta, \phi)=\frac{1}{2 f}\left[A+C+\sqrt{(A-C)^{2}+B^{2}}\right] .
\end{aligned}
$$

Jarrett et al. (2003) find that LGA galaxies are well fitted, outside their central nucleus or core, by a Sérsic profile,

$$
\mu(X)=\mu_{d} \exp \left[-(X / \alpha)^{1 / n}\right]
$$

The functional form adopted by Jarrett et al. (2003), in which $\alpha$ is a scale radius, is readily converted to the more usual form,

$$
\mu(X)=\mu\left(R_{e}\right) \exp \left\{-b_{n}\left[\left(X / R_{e}\right)^{1 / n}-1\right]\right\}
$$

in which $R_{e}$ is the effective radius, or half-light radius. The conversion from scale length $\alpha$ to effective radius $R_{e}$ is $R_{e}=\left(b_{n}\right)^{n} \alpha$, where an adequate approximation to $b_{n}$ is (Ciotti \& Bertin 1999)

$$
b_{n} \approx 2 n-\frac{1}{3}+\frac{4}{405 n}+\frac{46}{25515 n^{2}} .
$$

For the spiral galaxies in the LGA, the best-fitting profiles are usually close to exponential; $90 \%$ of the spiral galaxies have $0.7 \leq n \leq 1.4$ in the $K_{s}$ band. In this range of $n$, the effective radius ranges from $R_{e}=1.06 \alpha$ (when $n=0.7$ ) to $R_{e}=3.55 \alpha$ (when $n=1.4$ ). 
If a galaxy has a surface brightness profile given by equation (9), then if it were viewed face-on (that is, from along the $\theta=0$ axis) its profile would be

$$
\mu(X)=\mu_{d}^{0} \exp \left[-\left(X / \alpha^{0}\right)^{1 / n}\right]
$$

where $\alpha^{0}$ is the scale length along the apparent long axis and $\mu_{d}^{0}$ is the central surface brightness, as seen from the angle $\theta=0$. Let $R_{20}^{0}$ be the value of $X$ at which the surface brightness falls to the specified isophotal brightness $\mu_{20}=20 \mathrm{mag} \operatorname{arcsec}^{-2}$. For the profile of equation (12),

$$
R_{20}^{0}=\alpha^{0}\left[\ln \left(\mu_{d}^{0} / \mu_{20}\right)\right]^{n} .
$$

If we actually view the disk galaxy from an angle $(\theta, \phi)$, the central surface brightness of the disk is

$$
\mu_{d}(\theta, \phi)=\mu_{d}^{0} \beta \sqrt{D(\theta, \phi) E(\theta, \phi)}
$$

and the scale length along the apparent long axis is

$$
\alpha(\theta, \phi)=\alpha^{0} / \sqrt{D(\theta, \phi)} .
$$

The isophotal radius $R_{20}$ at which the surface brightness on the long axis falls to $\mu_{20}$ is

$$
R_{20}(\theta, \phi)=\alpha\left[\ln \left(\mu_{d} / \mu_{20}\right)\right]^{n} .
$$

The apparent axis ratio of the disk, seen from the angle $(\theta, \phi)$, is $q=(D / E)^{1 / 2}$. Thus, the face-on isophotal radius, $R_{20}^{0}$, is related to the observed values of $R_{20}(\theta, \phi)$ and $\alpha(\theta, \phi)$ by the relation

$$
R_{20}^{0}=\sqrt{D} R_{20}\left[1+\left(\alpha / R_{20}\right)^{1 / n} \ln (q / \beta D)\right]^{n} .
$$

Although $R_{20}, \alpha$, and $q$ can be measured for any particular galaxy seen in projection, $\beta$ and $D(\theta, \phi)$ cannot. However, if the disk is axisymmetric, then $\beta=D=1$, the scale length $\alpha$ is independent of viewing angle, and the face-on isophotal radius can be deduced from the observed properties:

$$
R_{20}^{0}=R_{20}\left[1+\left(\alpha / R_{20}\right)^{1 / n} \ln q\right]^{n}
$$

For all $n>0$, an axisymmetric disk appears smallest when seen face-on $\left(R_{20}^{0} \leq R_{20}\right)$.

\section{SHAPE OF AXISYMMETRIC DISKS}

If we assume that spiral galaxies are axisymmetric, then the analysis of $\S 2$ suggests a way of assembling an inclinationindependent subsample of disk galaxies from the 2MASS LGA. If the LGA contains all galaxies with $R_{20}>60^{\prime \prime}$, then, assuming axisymmetry, it will contain all galaxies with $R_{20}^{0}>60^{\prime \prime}$. Thus, from the tabulated values for $R_{20}, \alpha, n$, and $q$ (Jarrett et al. 2003), I compute $R_{20}^{0}$ for each spiral galaxy, using equation (18). If $R_{20}^{0}>60^{\prime \prime}$, the spiral is included in the inclination-independent subsample.

In this way, I find that 193 spiral galaxies in the 2MASS LGA have $R_{20}^{0}>60^{\prime \prime}$. Of these galaxies, 130 have a Hubble type earlier than Sc (14 S0/a, $6 \mathrm{Sa}, 28 \mathrm{Sab}, 40 \mathrm{Sb}$, and $42 \mathrm{Sbc}$ ); I call these galaxies the "early spirals." The remaining 63 spirals have a Hubble type of Sc or later (36 Sc, $18 \mathrm{Scd}, 8 \mathrm{Sd}$, and $1 \mathrm{Sm}$ ); I call these the "late spirals." If I had not done the inclination cor-

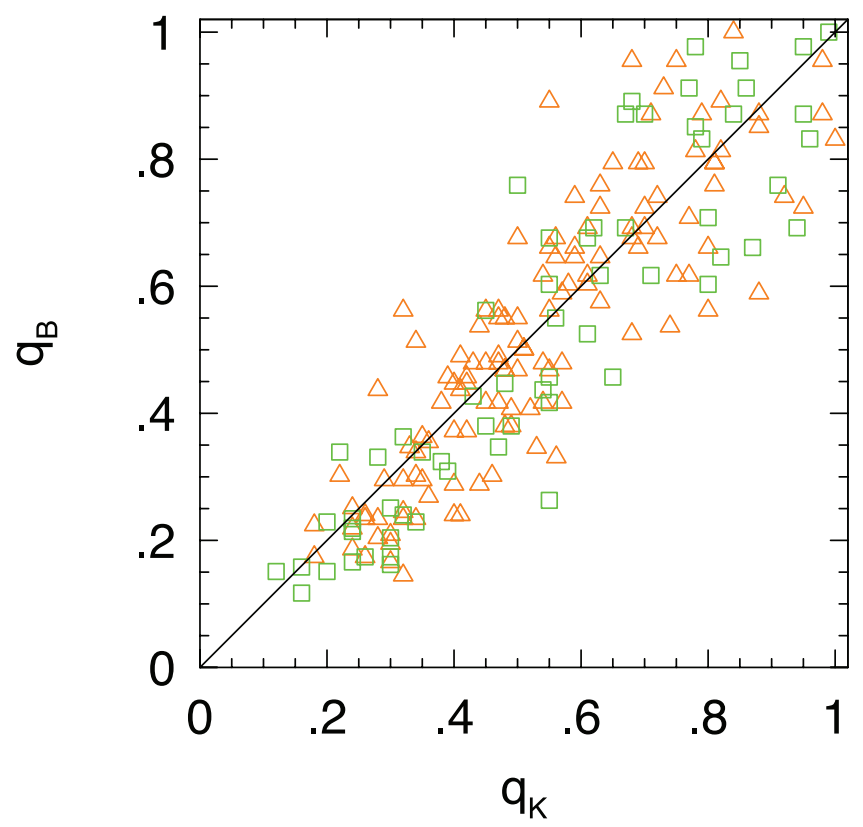

FIG. 1.-Axis ratio of the $25 \mathrm{mag} \mathrm{arcsec}^{-2}$ isophote in the $B$ band vs. the axis ratio of the $3 \sigma$ isophote in the $K_{s}$ band. Squares indicate late spirals (Sc or later); triangles indicate early spirals.

rection to find $R_{20}^{0}$, I would have found 161 early spirals and 117 late spirals with $R_{20}>60^{\prime \prime}$.

The apparent axis ratios $q_{K}$ and $q_{B}$ for the 193 LGA spirals in the inclination-corrected sample are shown in Figure 1. Spirals with $q_{B} \lesssim 0.3$ are generally less flat in the $K_{S}$ band than in the $B$ band. It can also been seen that extremely flat spirals, with $\left(q_{B}+q_{K}\right) / 2<0.2$, and nearly round spirals, with $\left(q_{B}+q_{K}\right) /$ $2>0.9$, are more likely to be late types (Fig. 1, squares) than early types (Fig. 1, triangles). Intermediate axis ratios, however, with $\left(q_{B}+q_{K}\right) / 2 \sim 0.5$, are strongly dominated by early spirals. When galaxies with detectable visible-light bars (types SAB and $\mathrm{SB}$ ) are compared to galaxies of type $\mathrm{S}$ and SA, no significant difference is found in their axis ratio distributions; $P \geq 0.1$ as measured both by a Kolmogorov-Smirnov test and by a $\chi^{2}$ test, in both bands.

On a cautionary note, it should be pointed out that $q_{K}$, the axis ratio of the $3 \sigma$ isophote, does not measure the shape at the same physical distance from each galaxy's center. Because looking at a disk edge-on enhances the surface brightness, the distance $R_{3 \sigma}$, the semimajor axis of the $3 \sigma$ isophote, will be larger when a galaxy is seen edge-on than when it is seen face-on. Thus, $q_{K}$ measures the shape at larger radii, on average, for galaxies with small $q_{K}$ than for galaxies with large $q_{K}$. To illustrate the trend, Figure 2 shows $R_{3 \sigma}$, in units of the effective radius, $R_{e}=\left(b_{n}\right)^{n} \alpha$, as a function of $\ln q_{K}$. On average, $R_{3} / R_{\mathrm{e}}$ tends to increase as $q_{K}$ decreases. The best linear fit to the data for late spirals, shown as the dashed line in Figure 2, is

$$
R_{3 \sigma} / R_{e}=0.976-0.617 \ln q_{K}
$$

with a correlation coefficient $r=-0.64$. The best fit to the data for early spirals, shown as the dotted line in Figure 2, is

$$
R_{3 \sigma} / R_{e}=1.235-0.670 \ln q_{K}
$$

with a correlation coefficient $r=-0.56$. Although the scatter in the relation is large, the $3 \sigma$ isophote tends to be closer to the 


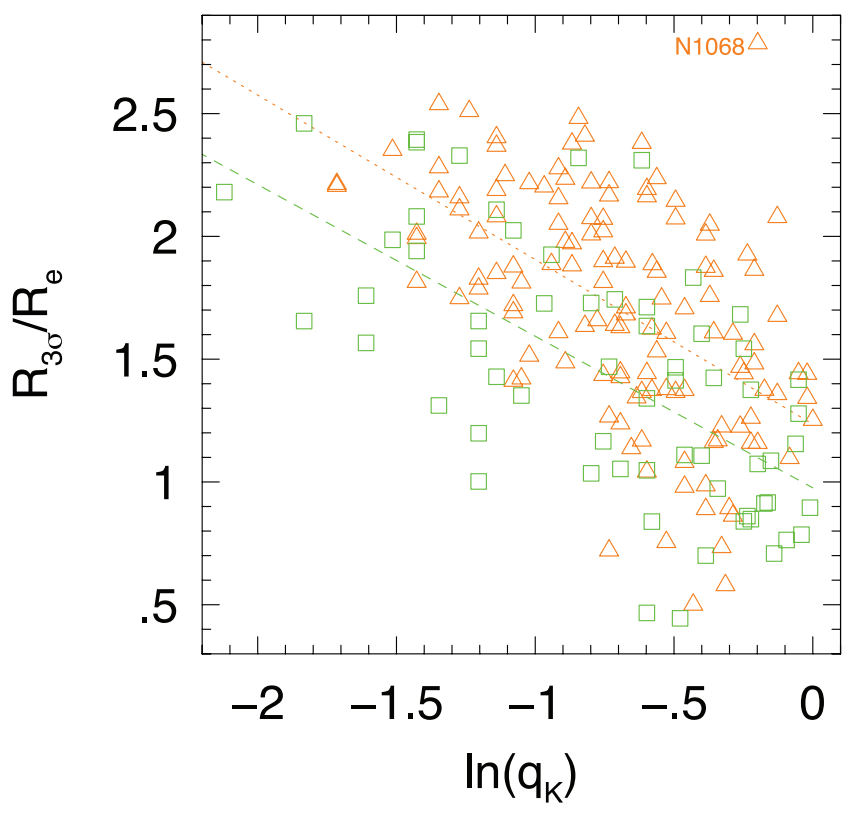

FIG. 2.- Semimajor axis $R_{3} \sigma$ of the $3 \sigma$ isophote, measured in units of the half-light radius $R_{e}$ of the best Sersic profile, plotted as a function of the axis ratio $q_{K}$. Squares indicate late spirals; the dashed line is the best fit to their $R_{3 \sigma} / R_{e}$ vs. $\ln q_{K}$ relation. Triangles indicate early spirals; the dotted line is the best fit to their $R_{3}{ }_{\sigma} / R_{e}$ vs. $\ln q_{K}$ relation. The labeled outlier, NGC 1068 , is excluded from the fit.

galaxy's center for nearly face-on axisymmetric disks than for nearly edge-on disks. If the isoluminosity surfaces of a disk galaxy were perfectly similar oblate spheroids, it would not matter where $q_{K}$ is measured. However, for early-type, big-bulged spirals, the value of $q_{K}$ measured for galaxies of low inclination can be significantly affected by light from the galaxy's central bulge. A similar relation holds true between $R_{25}$ and $q_{B}$, the apparent axis ratio of the $\mu_{B}=25 \mathrm{mag} \operatorname{arcsec}^{-2}$. However, since $R_{25}$ in the $B$ band is typically larger than $R_{3} \sigma$ in the $K_{S}$ band, the value of $q_{B}$ is usually not strongly influenced by bulge light. For the early spirals, the mean and standard deviation of $R_{25} / R_{3} \sigma$ are $1.90 \pm 0.62$; for the late spirals, $R_{25} / R_{3}=2.45 \pm 1.04$. (For two early spirals at low Galactic latitude, WKK 4748 and Maffei 2, $R_{25} / R_{3 \sigma} \lesssim 0.5$.)

\subsection{Nonparametric Fits}

The distributions of apparent axis ratios, $f\left(q_{K}\right)$ and $f\left(q_{B}\right)$, were estimated for the galaxies in the sample using a nonparametric kernel technique (Vio et al. 1994; Tremblay \& Merritt 1995; Ryden 1996). Estimating a function $f$ by the use of kernels gives a smooth estimate function $\hat{f}$ with no a priori restrictions on its shape, unlike a parametric fit to $f$. Given a sample of $n$ axis ratios, $q_{1}, q_{2}, \ldots, q_{n}$, the kernel estimate of the frequency distribution $f(q)$ is

$$
\hat{f}(q)=\frac{1}{n} \sum_{i=1}^{n} \frac{1}{h_{i}} K\left(\frac{q-q_{i}}{h_{i}}\right),
$$

where $K(x)$ is the kernel function, normalized so that its integral over all $x$ is equal to 1 . To ensure that the estimate $\hat{f}$ is smoothly differentiable, I adopt a Gaussian kernel, $K(x) \propto \exp \left(-x^{2} / 2\right)$. The parameter $h_{i}$ in equation (21) is the kernel width, determined using the standard adaptive two-stage estimator of Abramson (1982). In the two-stage estimator, the first stage consists of making an initial estimate $\hat{f}_{0}$ using a fixed kernel width $h$. The
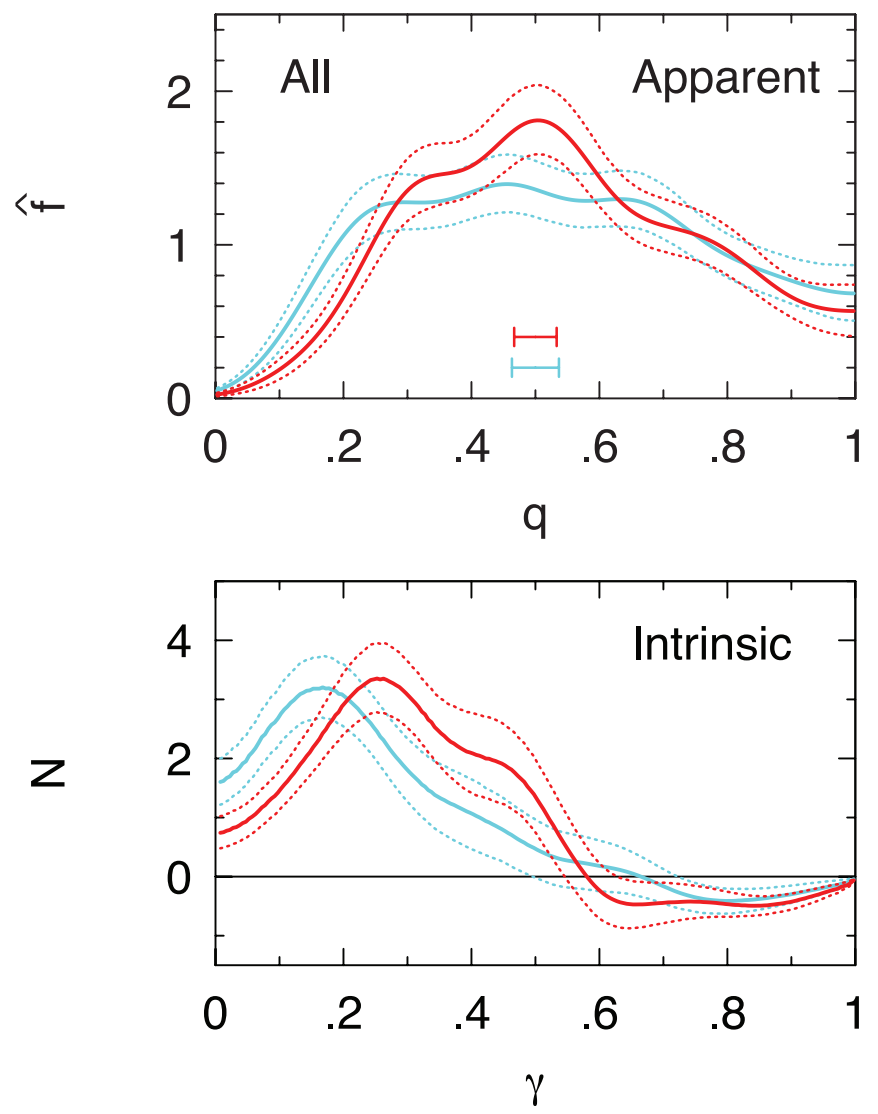

FIG. 3.-Top: Distribution of apparent axis ratios for the inclination-adjusted sample of 2MASS LGA spirals. Red lines indicate the $K_{s}$ axis ratios; blue lines indicate the $B$ axis ratios. The solid line in each case is the best fit, while the dotted lines indicate the $80 \%$ confidence interval estimated from bootstrap resampling. The horizontal error bars show the initial kernel width $h$. Bottom: Distribution of intrinsic axis ratios, assuming the spirals are randomly oriented oblate spheroids.

initial kernel width is given by the formula $h=0.9 A n^{-0.2}$, with $A=\min \left(\sigma, Q_{4} / 1.34\right)$, where $\sigma$ is the standard deviation of the axis ratios and $Q_{4}$ is the interquartile range. For samples that are not extremely skewed, this formula minimizes the mean square error of the estimate (Silverman 1986; Vio et al. 1994). The second stage consists of making the final estimate $\hat{f}$, using at each data point $q_{i}$ the kernel width

$$
h_{i}=h\left[\frac{G}{\hat{f}_{0}\left(q_{i}\right)}\right]^{1 / 2},
$$

where $G$ is the geometric mean of $\hat{f}_{0}$ over all values of $q_{i}$.

The estimated distribution of axis ratios for the 193 spirals in the inclination-corrected sample is shown in Figure 3 (top). The solid red line indicates $\hat{f}\left(q_{K}\right)$, while the solid blue line indicates $\hat{f}\left(q_{B}\right)$. The dotted lines indicate the $80 \%$ error intervals estimated by bootstrap resampling. In the bootstrap analysis, I randomly selected $n=193$ data points, with substitution, from the original sample of 193 axis ratios; a new estimate $\hat{f}$ was then created from the bootstrapped data. After doing 5000 bootstrap estimates, I determined the $80 \%$ error interval; in Figure 3, 10\% of the bootstrap estimates lie above the upper dotted line, and $10 \%$ lie below the lower dotted line. If the spiral galaxies were infinitesimally thin, perfectly circular disks, the distribution function would be $f=1$ for $0 \leq q \leq 1$; the deviations of $f\left(q_{K}\right)$ and $f\left(q_{B}\right)$ from this form reveal that the spiral galaxies in our 
sample are neither infinitesimally thin nor perfectly circular. In the $K_{S}$ band, galaxies are slightly rounder on average than in the $B$ band: $\left\langle q_{K}\right\rangle=0.538$ versus $\left\langle q_{B}\right\rangle=0.525$. In addition, there is a slightly greater spread in shapes in the $B$ band: $\sigma\left(q_{K}\right)=0.212$ versus $\sigma\left(q_{B}\right)=0.234$.

Given the assumption of oblateness, it is straightforward to do a numerical inversion of $\hat{f}(q)$, the estimated distribution of apparent axis ratios, to find $\hat{N}(\gamma)$, the estimated distribution of intrinsic axis ratios. The relation between $\hat{f}(q)$ and $\hat{N}(\gamma)$ is

$$
\hat{f}(q)=\int_{0}^{q} P(q \mid \gamma) \hat{N}(\gamma) d \gamma
$$

where $P(q \mid \gamma) d q$ is the probability that a galaxy with a shortto-long axis ratio $\gamma$ has an apparent axis ratio in the range $q \rightarrow q+d q$ when seen from a randomly chosen viewing angle. For a population of oblate spheroids (Sandage et al. 1970)

$$
P(q \mid \gamma)= \begin{cases}\frac{q}{\left(1-\gamma^{2}\right)^{1 / 2}\left(q^{2}-\gamma^{2}\right)^{1 / 2}} & \text { if } \gamma \leq q \leq 1 \\ 0 & \text { otherwise. }\end{cases}
$$

Equation (23) is a Volterra equation of the first kind; in its discretized form, it can be inverted to find $\hat{N}$ by a process of forward substitution (see Vincent \& Ryden [2005] for details).

Figure 3 (bottom) shows the distributions of intrinsic axis ratios in the $K_{s}$ band (red line) and the $B$ band (blue line). The solid line, in each case, is the distribution found by inverting equation (23). Because $\hat{N}$ is a deconvolution of $\hat{f}$, any noise present in $\hat{f}$ will be amplified in $\hat{N}$; to produce an acceptably smooth estimate of $N$, it is thus necessary to use a value of the initial kernel width $h$ that is larger than the optimal value for computing $\hat{f}$ (Tremblay \& Merritt 1995). For computing $\hat{N}$, I used a kernel width $h$ that was $20 \%$ greater than the value used to compute $\hat{f}$.

The deduced distributions $\hat{N}$ show that the most probable value of $\gamma$ is $\gamma_{K}=0.25$ in the $K_{s}$ band and $\gamma_{B}=0.17$ in the $B$ band. A striking aspect of the distributions $\hat{N}$ seen in Figure 3 is that they are negative for large values of $\gamma$. In the $K_{s}$ band, $\hat{N}$ dips below zero for $\gamma_{K}>0.58$; in the $B$ band, $\hat{N}$ is below zero for $\gamma_{B}>0.67$. This is a physical absurdity, implying a negative number of nearly spherical galaxies. The $80 \%$ confidence band, delineated by the dotted lines in Figure 3 (bottom), falls below zero in both bands for $\gamma \geq 0.7$. This means that fewer than $10 \%$ of the bootstrap resamplings yield nonnegative estimates in this range of $\gamma$. The hypothesis that the spiral galaxies are a population of randomly oriented oblate spheroids can thus be rejected at the $90 \%$ (one sided) confidence level. If the $98 \%$ confidence band is laid out in a similar manner, with $1 \%$ of the bootstrap resamplings lying above the band and $1 \%$ lying below, it is found that in the $K_{s}$ band, the confidence band dips below zero for large $\gamma$. Thus, in the $K_{s}$ band, the oblate hypothesis can be rejected at the $99 \%$ (one sided) confidence level. In the $B$ band, where the shape is measured farther from the galaxies' centers, the oblate hypothesis cannot be rejected at the $99 \%$ confidence level.

For the complete inclination-adjusted sample, the hypothesis that the galaxies are perfectly axisymmetric can be rejected at a fairly strong confidence level, particularly in the near-infrared. However, Figure 1 reveals that the distribution of apparent shapes is different for late spirals and for early spirals. Thus, it is worthwhile to look at $\hat{f}(q)$ and the derived $\hat{N}(\gamma)$ for late and early spirals separately. Figure 4 (top) shows $\hat{f}\left(q_{K}\right)$ and $\hat{f}\left(q_{B}\right)$ for the $n=63$ late spirals in the sample. The distribution of apparent axis ratios
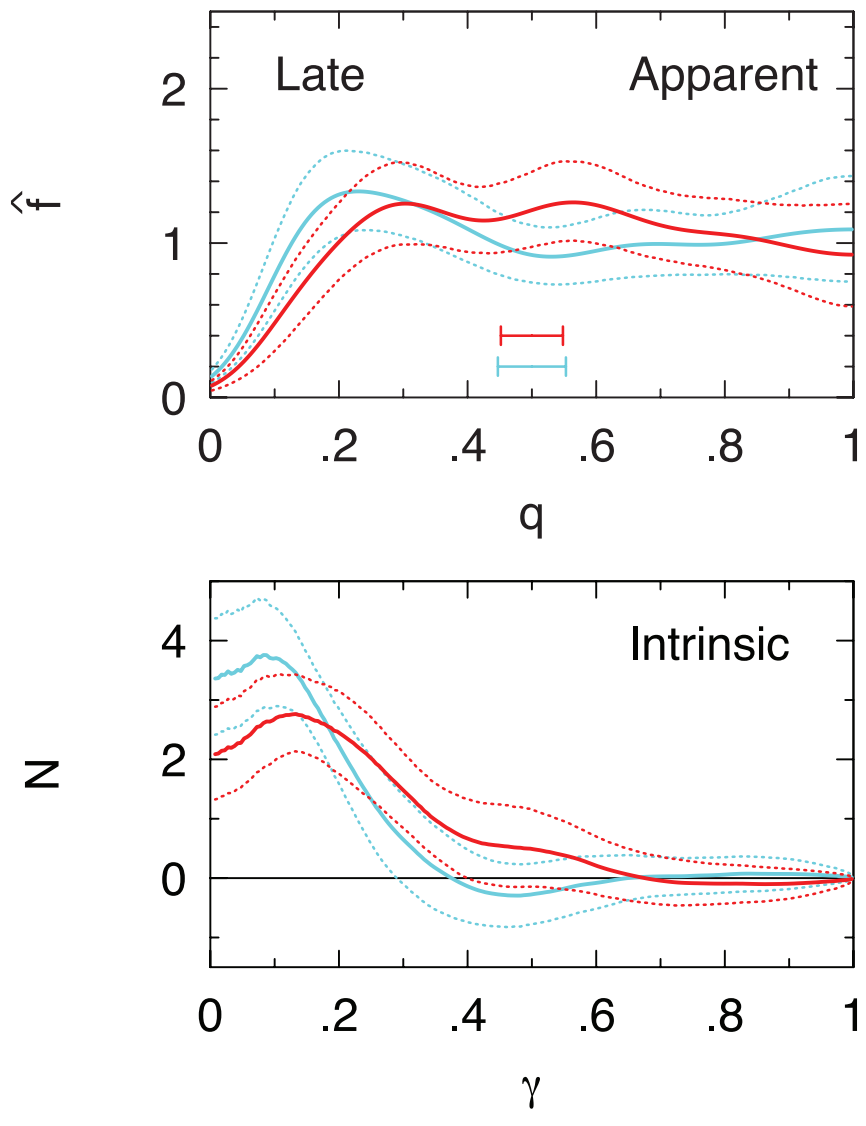

FIG. 4.-Same as Fig. 3, but including only the $n=63$ spirals of Hubble type Sc and later.

in the $K_{S}$ band, indicated by the red lines, is similar to the distribution in the $B$ band, indicated by the blue lines. In both cases, the distribution $\hat{f}$ is nearly constant over a wide range of $q$. The deduced distributions of intrinsic axis ratios, $\hat{N}\left(\gamma_{K}\right)$ and $\hat{N}\left(\gamma_{B}\right)$, are indicated by the red and blue lines, respectively, in Figure 4 (bottom). In both bands, the distribution $\hat{N}$ never falls below zero; thus, the oblate hypothesis is perfectly acceptable, both for the $B$ band data and for the $K_{s}$ band data. If the oblate hypothesis is accepted, the average disk thickness is smaller in the $B$ band: $\left\langle\gamma_{B}\right\rangle=0.12$ as compared to $\left\langle\gamma_{K}\right\rangle=0.19$. In both bands, the width of the function $\hat{N}$ is comparable to the kernel width $h$ used, indicating that the true spread in $\gamma_{B}$ and $\gamma_{K}$ may be less than is shown in the smoothed distributions of Figure 4.

The apparent shapes of early spirals, shown in Figure 5 (top), are strikingly different from the apparent shapes of late spirals. For early spirals, the distribution $\hat{f}$ is not uniform over $q$, but instead is peaked at $q \sim 0.5$, with a marked scarcity of galaxies with $q \gtrsim 0.8$. Such a nonuniform distribution is typical of a population of triaxial ellipsoids. The distribution of $q_{K}$ (Fig. 5, red lines) is particularly strongly peaked at $q \sim 0.5$, which may be attributed, in part, to a contribution of light from nonaxisymmetric bulges. However, even the distribution of $q_{B}$, measured at a much larger radius within each galaxy, is inconsistent with axisymmetry. The deduced distributions of intrinsic axis ratios, shown in Figure 5 (bottom), demonstrate how grossly inconsistent the observed distributions of $q_{K}$ and $q_{B}$ are with the hypothesis of randomly oriented oblate spheroids. The oblate hypothesis can, in fact, be ruled out at the $99 \%$ confidence level for both the $K_{s}$ band isophotal shapes and the $B$-band isophotal shapes. Thus, the deviations from axisymmetry in the full sample of spiral 

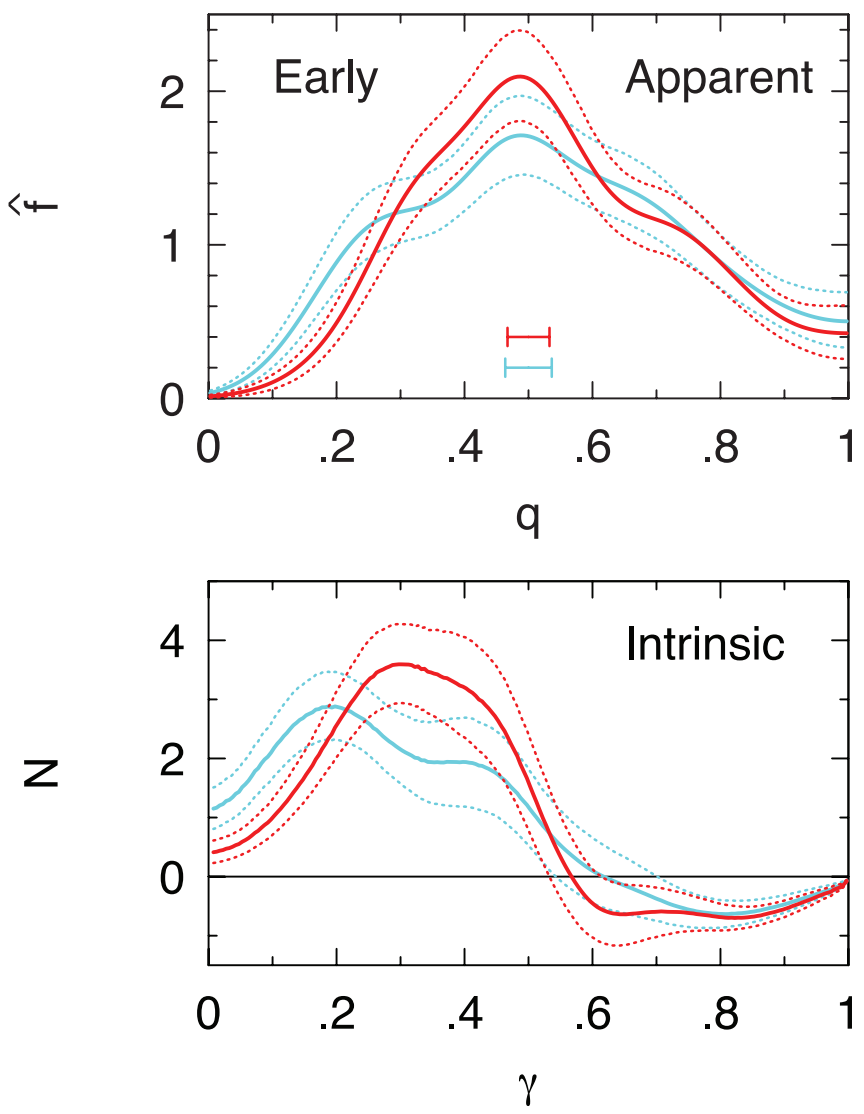

FIG. 5.-Same as Fig. 3, but including only the $n=130$ spirals of Hubble type Sbc and earlier.

galaxies can be attributed to the early spirals in the sample; the late spirals are perfectly consistent with axisymmetry.

\subsection{Parametric Fits}

For comparison with previous studies, it is useful to make parametric estimates of $N(\gamma)$, in addition to the nonparametric kernel estimates of $\S 3.1$. A common convention (Huizinga \& van Albada 1992; Fasano et al. 1993) is to fit the distribution of intrinsic axis ratios with a Gaussian:

$$
N(\gamma) d \gamma \propto \exp \left[-\frac{\left(\gamma-\mu_{\gamma}\right)^{2}}{2 \sigma_{\gamma}^{2}}\right] d \gamma
$$

for $0 \leq \gamma \leq 1$, with $N=0$ elsewhere. Inspection of Figure 4 (bottom) indicates that this should provide a reasonable fit for the late spirals, at least.

The best-fitting values of $\mu_{\gamma}$ and $\sigma_{\gamma}$ were determined by a $\chi^{2}$ fit to the binned distribution of $q_{K}$ and $q_{B}$. In each band, the $n$ values of $q$ were divided into $n_{\text {bin }}=12$ bins of equal width. After choosing particular values for $\mu_{\gamma}$ and $\sigma_{\gamma}$, I randomly chose $n$ values of $\gamma$, drawn from the distribution of equation (25). After randomly selecting a viewing angle for each mock disk, I computed its apparent axis ratio $q(\gamma, \theta, \phi)$ from the formulas in $\S 2$. The model axis ratios were then binned in the same way as the observed axis ratios. After repeating this procedure 8000 times for a given $\left(\mu_{\gamma}, \sigma_{\gamma}\right)$ pair, I calculated the mean and standard deviation of the expected number of model galaxies in each bin and computed a $\chi^{2}$ probability for that particular pair of parameters.

Figure 6 shows the isoprobability contours in the twodimensional parameter space, where the probability is measured

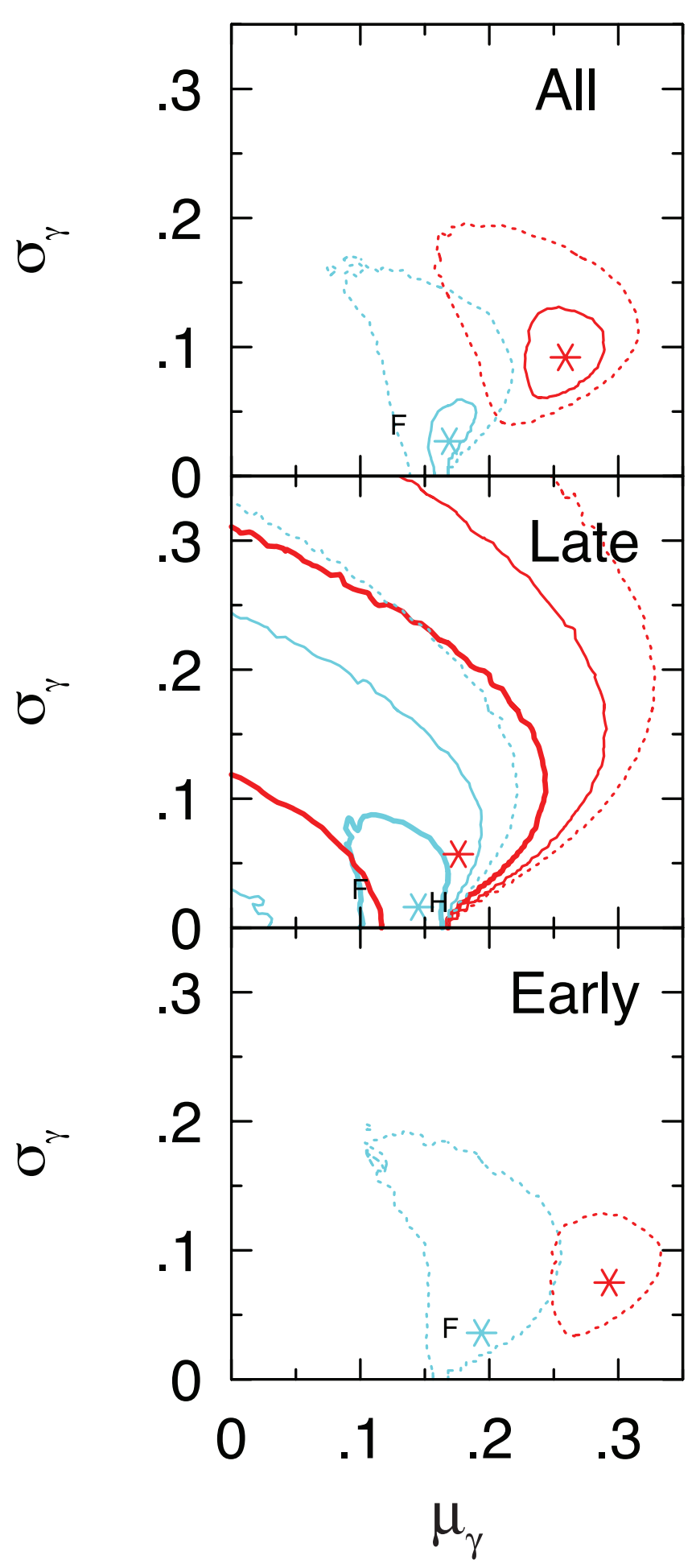

FIG. 6.-Top: Asterisks indicate the best-fitting values of $\mu_{\gamma}$ and $\sigma_{\gamma}$ to the complete sample of LGA spirals; the heavy solid line, light solid line, and dotted line indicate the $P=0.5,0.1$, and 0.01 isoprobability contours, found by a $\chi^{2}$ fit. Red indicates shapes deduced from $K_{s}$ data; blue indicates shapes deduced from $B$ data. Middle: Same as top, but for spirals of Hubble type Sc or later. Bottom: Same as top and bottom, but for spirals of type Sbc or earlier. F indicates fits of Fasano et al. (1993); H indicates fit of Huizinga \& van Albada (1992) for Sc spirals. 
by a $\chi^{2}$ fit to the binned data. The fit to the $K_{s}$-band data is shown by the red lines; the fit to the $B$-band data is shown by the blue lines. In each band, the best-fitting values of $\mu_{\gamma}$ and $\sigma_{\gamma}$ are indicated by an asterisk.

In Figure 6 (top), the fit is made to the complete inclinationadjusted sample of $n=193$ spirals. In the $K_{s}$ band, the best fit in this region of parameter space is $\left(\mu_{\gamma}, \sigma_{\gamma}\right)=(0.26,0.09)$, which yields a $\chi^{2}$ probability $P \approx 0.20$. In the $B$ band, the best fit is $\left(\mu_{\gamma}, \sigma_{\gamma}\right)=(0.17,0.03)$, which yields $P \approx 0.17$. For these fits, the biggest contributions to $\chi^{2}$ come from the two bins with $q \geq 0.83$, for which this axisymmetric model predicts more galaxies than are observed. In the $K_{S}$ band, spiral galaxies are not only thicker, on average, than in the $B$ band, but they also have a greater spread in thicknesses. The typical $\gamma$-value I find for spirals in the $B$ band, $\mu_{\gamma}=0.17$, is greater than the value of $\mu_{\gamma}=$ 0.13 found by Fasano et al. (1993) for a volume-limited sample of 766 spirals, using apparent axis ratios taken from the Third Reference Catalogue of Bright Galaxies (de Vaucouleurs et al. 1991). As seen in Figure 6 (top), the best values of $\left(\mu_{\gamma}, \sigma_{\gamma}\right)$ found by Fasano et al. (1993) lie just inside the $P=0.01$ contour for the 2MASS LGA spirals.

Figure 6 (middle) shows the fit for the late spirals only (Hubble type Sc and later). The $n=63$ galaxies in this subsample do not place a strong constraint on $\mu_{\gamma}$ and $\sigma_{\gamma}$; the $P=0.5$ contour, indicated by the heavy solid line, contains a large swath of parameter space, particularly in the $K_{S}$ band. The best fit in the $K_{s}$ band is $\left(\mu_{\gamma}, \sigma_{\gamma}\right)=(0.18,0.06)$, which yields a $\chi^{2}$ probability $P \approx 0.91$. In the $B$ band, the best fit is $\left(\mu_{\gamma}, \sigma_{\gamma}\right)=(0.14,0.02)$, yielding $P \approx 0.80$. Once again, as for the complete sample, the $K_{s}$-band data indicate both a larger average thickness and a greater spread in thicknesses than do the $B$ data. However, in both bands, the data are consistent with $\sigma_{\gamma}=0$. An excellent fit results if all the late spirals are assumed to have an intrinsic axis ratio $\gamma$ equal to, or slightly smaller than, the smallest apparent axis ratio $q$ in the subsample $\left(q_{K}=0.12\right.$ for NGC 4302 and $q_{B}=0.12$ for NGC 5907). The best fit found by Huizinga \& van Albada (1992) for a sample of Sc galaxies, $\left(\mu_{\gamma}, \sigma_{\gamma}\right)=(0.16,0.02)$, and by Fasano et al. (1993) for a sample of galaxies of type $4.5<t \leq 7.0$, $\left(\mu_{\gamma}, \sigma_{\gamma}\right)=(0.10,0.03)$, are both statistically consistent with the results for late-type LGA 2 MASS spirals in the $B$ band; see Figure 6 (middle).

Figure 6 (bottom) shows the results of fitting a Gaussian distribution for $\gamma$ to the early spirals (Hubble type Sbc and earlier). The best fits are not as good as the excellent fits found for the late spirals. The best fit in the $K_{s}$ band is $\left(\mu_{\gamma}, \sigma_{\gamma}\right)=(0.29,0.08)$, for which the $\chi^{2}$ probability is $P \approx 0.06$. The best fit in the $B$ band is $\left(\mu_{\gamma}, \sigma_{\gamma}\right)=(0.20,0.04)$, yielding $P \approx 0.08$. The relatively poor fits are the result of demanding axisymmetry; the Gaussian form of equation (25) is not primarily to blame, since the nonparametric kernel estimate revealed that no distribution $N(\gamma)>0$ gives a good fit to the early spirals. As with the late spirals, the average thickness and the spread of thicknesses is greater in the $K_{s}$ band than in the $B$ band. The greater disk thickness in the infrared is more pronounced for the early spirals than for the late spirals. For early spirals, the best-fitting $\mu_{\gamma}$ in the $K_{s}$ band is $50 \%$ greater than in the $B$ band; for late spirals, the best-fitting $\mu_{\gamma}$ in the $K_{s}$ band is only $20 \%$ greater than in the $B$ band.

\section{SHAPE OF NONAXISYMMETRIC DISKS}

Given that a distribution of axisymmetric $(\epsilon=0)$ disks does not give a good fit to early spirals, it would be useful to know which distribution of nonaxisymmetric disks gives the best fit to the early spirals (and to the late spirals, for that matter). A fundamental difficulty is that even if $f(q)$ were known perfectly, it would not uniquely determine $N(\gamma, \epsilon)$. However, the joint distribution of the disk thickness $\gamma$ and the disk ellipticity $\epsilon$ is not beyond all conjecture. I use the approach of assuming a parameterized functional form for the distribution $N(\gamma, \epsilon)$ and finding which values of the parameters give the best fit to the observed distributions of axis ratios. This approach yields permissible fits to $N(\gamma, \epsilon)$, but these fits, it should be remembered, are not unique solutions.

\subsection{Gaussian Parametric Fits}

I assume that $N(\gamma, \epsilon)=N_{\gamma}(\gamma) N_{\epsilon}(\epsilon)$, where $N_{\gamma}$ is the same Gaussian assumed in equation (25) and $N_{\epsilon}$ is a Gaussian distribution (Lambas et al. 1992; Huizinga \& van Albada 1992; Fasano et al. 1993; Ryden 2004):

$$
N_{\epsilon}(\epsilon) d \epsilon \propto \exp \left[-\frac{\left(\epsilon-\mu_{\epsilon}\right)^{2}}{2 \sigma_{\epsilon}^{2}}\right] d \epsilon .
$$

The function $N(\gamma, \epsilon)$ is nonzero inside the region $0 \leq \gamma \leq 1$ and $0 \leq \epsilon \leq 1-\gamma$. A complication of fitting nonaxisymmetric models is the increased difficulty of deriving an inclination-corrected subset of spiral galaxies. If a galaxy is not an axisymmetric disk, it is impossible from photometry alone to determine absolutely whether or not its face-on semimajor axis $R_{20}^{0}$ is greater than the cutoff value of $60^{\prime \prime}$. To illustrate the effects of nonaxisymmetry, consider a mildly triaxial disk, with $\epsilon \ll 1$. For such a disk, $\beta=$ $1-\epsilon, D \approx 1+2 \epsilon \cos ^{2} \phi$, and the face-on semimajor axis is, from equation (17),

$$
R_{20}^{0} \approx\left(1+\epsilon \cos ^{2} \phi\right) R_{20}\left[1+\left(\alpha / R_{20}\right)^{1 / n}(\ln q-\epsilon \cos 2 \phi)\right]^{n} .
$$

Thus, the face-on isophotal radius $R_{20}^{0}$ of a triaxial disk may be either smaller or larger than that of an axisymmetric disk with the same observed values of $R_{20}, \alpha, n$, and $q$. Since $R_{20}^{0}$ can be greater than the observed $R_{20}$ for a nonaxisymmetric disk, a sample complete to $R_{20}=60^{\prime \prime}$ is not guaranteed to be complete to $R_{20}^{0}=60^{\prime \prime}$. However, for mildly eccentric disks $(\epsilon \ll 1)$, the potential incompleteness effects should be small.

To make the inclination correction, I begin by assuming values for the parameters $\mu_{\gamma}, \sigma_{\gamma}, \mu_{\epsilon}$, and $\sigma_{\epsilon}$. I randomly select a value of $\gamma$ and of $\epsilon$ from the appropriate distribution functions and randomly choose a viewing angle $(\theta, \phi)$. I then compute $\beta=1-\epsilon, D(\theta, \phi)$, using equation $(7), E(\theta, \phi)$, using equation (8), and the apparent axis ratio $q=(D / E)^{1 / 2}$. I then have one possible $(\beta, D)$ pair corresponding to the observable apparent axis ratio $q$. Having binned the entire range of $q$, from $q=0$ to $q=1$, into 100 equal-width bins, I can then place the $(\beta, D)$ pair into the appropriate bin. I repeat the process of randomly choosing $\gamma, \epsilon, \theta$, and $\phi$, computing $\beta, D, E$, and $q$, and putting the resulting $(\beta, D)$ pair into the appropriate bin in $q$, until I have done a total of $\sim 5 \times 10^{6}$ iterations of this process, keeping a maximum of 1000 $(\beta, D)$ pairs for each bin in $q$. Now consider a specific galaxy with measured values of $R_{20}, \alpha, n$, and $q_{K}$. I go to the appropriate bin in axis ratio $q$ and pull out $(\beta, D)$ pairs, determining how many of the pairs result in $R_{20}^{0}>60^{\prime \prime}$, using equation (17). A fraction $F$ of the $(\beta, D)$ pairs, where $0 \leq F \leq 1$, will result in the face-on isophotal radius $R_{20}^{0}$ of this galaxy being greater than the lower limit of $60^{\prime \prime}$. I then created binned distributions of $q_{K}$ and $q_{B}$, with $n_{\text {bin }}=12$, for comparison with model distributions. In the binned distributions of observed axis ratios, the axis ratio for each galaxy is given a weight equal to the fraction $F$. As a consequence, the total number $n=\sum F$ of galaxies in the 
TABLE 1

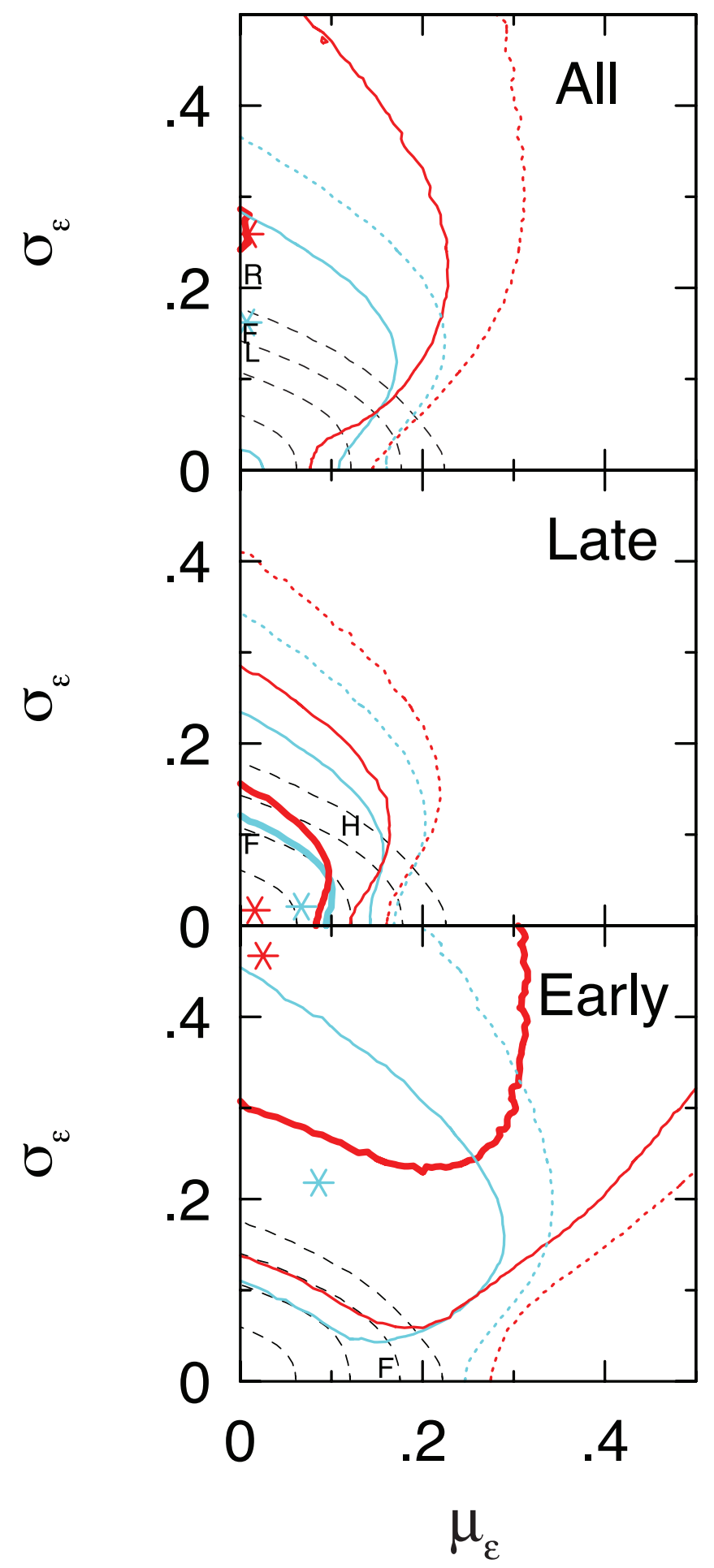

FIG. 7.-Top: Asterisks indicate the best-fitting values of $\mu_{\epsilon}$ and $\sigma_{\epsilon}$ to the complete sample of LGA spirals. The heavy solid line, light solid line, and dotted line indicate the $P=0.5,0.1$, and 0.01 isoprobability contours. Red indicates shapes from $K_{s}$ data; blue indicates shapes from $B$ data. Dashed lines show scatter in the Tully-Fisher relation (see $\S 5$ ); contours are drawn, starting at the lower left, at $0.25,0.5,0.75$, and $1.0 \mathrm{mag}$. Middle: Same as top, but for spirals of Hubble type Sc or later. Bottom: Same as top and bottom, but for spirals of type Sbc or earlier. F indicates Fasano et al. (1993), L indicates Lambas et al. (1992), R indicates Ryden (2004), and H indicates Huizinga \& van Albada (1992).
Best Parametric Fits

\begin{tabular}{|c|c|c|c|c|c|c|}
\hline Band & $\mu_{\gamma}$ & $\sigma_{\gamma}$ & $\mu_{\epsilon}$ & $\sigma_{\epsilon}$ & $\mu_{\eta}$ & $\sigma_{\eta}$ \\
\hline \multicolumn{7}{|c|}{ All Sample } \\
\hline$K_{s} \ldots \ldots \ldots \ldots \ldots \ldots$ & 0.26 & 0.09 & 0.01 & 0.26 & -0.14 & 2.74 \\
\hline$B \ldots \ldots \ldots \ldots \ldots \ldots \ldots \ldots$ & 0.17 & 0.03 & 0.01 & 0.16 & -2.36 & 0.96 \\
\hline \multicolumn{7}{|c|}{ Late Sample } \\
\hline$K_{s} \ldots \ldots \ldots \ldots \ldots$ & 0.18 & 0.06 & 0.02 & 0.02 & -3.86 & 0.74 \\
\hline$B \ldots \ldots \ldots \ldots \ldots \ldots \ldots$ & 0.14 & 0.02 & 0.07 & 0.02 & -2.63 & 0.13 \\
\hline \multicolumn{7}{|c|}{ Early Sample } \\
\hline$K_{s} \ldots \ldots \ldots \ldots \ldots \ldots$ & 0.29 & 0.08 & 0.02 & 0.47 & -0.14 & 1.74 \\
\hline$B$ & 0.20 & 0.04 & 0.09 & 0.22 & -1.77 & 0.83 \\
\hline
\end{tabular}

binned distribution may be nonintegral. For convenience, I round the number $n$ to the nearest integer by multiplying the number of galaxies per bin by the factor $C=\operatorname{int}(n+0.5) / n$.

Finding the global best fit in the four-dimensional parameter space $\left(\mu_{\gamma}, \sigma_{\gamma}, \mu_{\epsilon}, \sigma_{\epsilon}\right)$ involves a tedious search. The search can be simplified by noting that for relatively thin, mildly elliptical disks, the values of $\mu_{\gamma}$ and $\sigma_{\gamma}$ are determined by looking at galaxies that are close to edge-on, with $q<0.5$; by contrast, the values of $\mu_{\epsilon}$ and $\sigma_{\epsilon}$ are fixed by looking at galaxies that are close to face-on, with $q>0.5$. Thus, the best-fitting values of $\mu_{\gamma}$ and $\sigma_{\gamma}$ that I found by assuming $\epsilon=0$ should not be greatly changed when $0<\epsilon \ll 1$. I therefore fix the values of $\mu_{\gamma}$ and $\sigma_{\gamma}$ for each sample of galaxies to be equal to those determined in $\S 3.2$, where galaxies were assumed to be axisymmetric. This reduces the parameter space to two dimensions. After fixing $\mu_{\gamma}$ and $\sigma_{\gamma}$, I choose values of $\mu_{\epsilon}$ and $\sigma_{\epsilon}$. I then randomly select $n$ values of $\gamma$ from the distribution of equation (25), $n$ values of $\epsilon$ from the distribution of equation (27), and $n$ random viewing angles. The apparent axis ratio $q(\gamma, \epsilon, \theta, \phi)$ for each of $n$ mock galaxies is computed from the formulas given in $\S 2$. The model axis ratios are then binned into $n_{\text {bin }}=12$ equal-width bins. After repeating this procedure $\sim 10^{4}$ times for a given $\left(\mu_{\epsilon}, \sigma_{\epsilon}\right)$ pair, I calculate the mean and standard deviation of the expected number of mock galaxies in each bin, and compute a $\chi^{2}$ probability for that particular set of parameters.

Figure 7 shows the isoprobability contours in $\left(\mu_{\epsilon}, \sigma_{\epsilon}\right)$ parameter space. The fit to the $K_{s}$-band data is shown by the red lines; the fit to the $B$-band data is shown by the blue lines. In each band, the best-fitting values of $\mu_{\epsilon}$ and $\sigma_{\epsilon}$ in the displayed region of parameter space are indicated by an asterisk. The bestfitting $\mu_{\epsilon}$ and $\sigma_{\epsilon}$ for each galaxy sample, at each wavelength, are given in Table 1. Figure 7 (top) displays the results for the complete sample of spirals, weighted by the appropriate fraction $F$ for each galaxy. The best fits have $\mu_{\epsilon} \approx 0$ and $\sigma_{\epsilon}=0.16$ ( $B$ band) and $\sigma_{\epsilon}=0.26$ ( $K_{s}$ band). The most probable ellipticity for these skewed distributions is $\epsilon \approx 0$, but there is a long tail to higher ellipticities. The results for the 2MASS LGA spirals are in good agreement with those of Lambas et al. (1992), Fasano et al. (1993), and Ryden (2004), who find good fits for Gaussian distributions with $\mu_{\epsilon}=0$, and $\sigma_{\epsilon} \sim 0.2$. [I only computed solutions with $\mu_{\epsilon} \geq 0$. It is mathematically permissible to have $\mu_{\epsilon}<0$. In the limit $-\mu_{\epsilon} \gg \sigma_{\epsilon}$, this leads to an exponential distribution function: $N_{\epsilon} \propto \exp \left(-\epsilon / h_{\epsilon}\right)$, with a scale length $h_{\epsilon}=\sigma_{\epsilon}^{2} /\left|\mu_{\epsilon}\right| \ll \sigma_{\epsilon}$. Exponential functions peaking at $\epsilon=0$ do not give a significantly better fit than Gaussian functions peaking at $\epsilon=0$.] 
Figure 7 (middle) shows the results of fitting a Gaussian distribution of ellipticities to the late spirals (Sc and later). This plot emphasizes that the apparent shapes of late spirals are consistent with axisymmetry. The best fit in each band has $\mu_{\epsilon} \leq 0.07$ and $\sigma_{\epsilon}=0.02$, but $\mu_{\epsilon}=0, \sigma_{\epsilon}=0$ also gives an excellent fit, with $P>0.5$. Although perfect axisymmetry is not compulsory, there are constraints on how large the disk ellipticities can be. The region in parameter space for which $P>0.5$ (demarcated by the heavy solid lines Fig. 7, middle) is confined within the limits $\mu_{\epsilon} \leq 0.10, \sigma_{\epsilon} \leq 0.15$. The parameters found by Fasano et al. (1993) for their sample of late-type galaxies, $\mu_{\epsilon}=0$ and $\sigma_{\epsilon}=0.09$, are in good agreement with our results. However, the parameters found by Huizinga \& van Albada (1992) for their sample of Sc galaxies, $\mu_{\epsilon}=0.12$ and $\sigma_{\epsilon}=0.11$, imply a greater mean ellipticity than is found in the 2MASS LGA sample.

Figure 7 (bottom) shows the results of fitting a Gaussian distribution of ellipticities to the early spirals (Sbc and earlier). The best-fitting values of $\sigma_{\epsilon}$ indicate a broad range of thicknesses: $\sigma_{\epsilon}=0.22$ in the $B$ band and $\sigma_{\epsilon}=0.47$ in the $K_{s}$ band. Although late spirals have excellent fits with $\sigma_{\epsilon}=0$, getting a good fit to the observed shapes of early spirals requires $\sigma_{\epsilon}>0$. With a broad tail stretching toward $\epsilon=1-\gamma$ (indicating a prolate shape), the best fit for early spirals in the $K_{s}$ band does not really represent a population of disks at all. If I quantify the triaxiality by the parameter $T \equiv\left(1-\beta^{2}\right) /\left(1-\gamma^{2}\right)$, the best fit in the $K_{s}$ band, $\left(\mu_{\gamma}, \sigma_{\gamma}, \mu_{\epsilon}, \sigma_{\epsilon}\right)=(0.29,0.08,0.02,0.27)$, produces a mean triaxiality parameter $\langle T\rangle=0.51$, closer to a prolate shape $(T=1)$ than an oblate shape $(T=0)$. The best fit to early spirals in the $B$ band also has a significant mean triaxiality, $\langle T\rangle=0.37$. For comparison, the mean triaxiality for the best fit to late spirals is $\langle T\rangle=0.04$ in the $K_{S}$ band and $\langle T\rangle=0.13$ in the $B$ band.

\subsection{Lognormal Parametric Fits}

Satisfactory fits to the distribution of apparent axis ratios can also be found using a lognormal distribution of ellipticities, rather than a Gaussian. If I define $\eta \equiv \ln \epsilon$, then a lognormal distribution can be written in the form

$$
\begin{aligned}
N_{\epsilon}(\epsilon) d \epsilon & \propto \exp \left[-\frac{\left(\eta-\mu_{\eta}\right)^{2}}{2 \sigma_{\eta}^{2}}\right] d \eta \\
& \propto \exp \left[-\frac{\left(\ln \epsilon-\mu_{\eta}\right)^{2}}{2 \sigma_{\eta}^{2}}\right] \frac{d \epsilon}{\epsilon}
\end{aligned}
$$

Motivation for the lognormal fit is provided by Andersen et al. (2001) and Andersen \& Bershady (2002), who find, by combining photometric and kinematic information for nearly face-on spirals, that a lognormal distribution provides a good fit to the observed disk ellipticities. Finding the best values of $\mu_{\eta}$ and $\sigma_{\eta}$ for each sample of spirals is done using the technique outlined in $\S 3.2$. Once again, the values of $\mu_{\gamma}$ and $\sigma_{\gamma}$ used were those that gave the best fit in the axisymmetric case.

Figure 8 displays the isoprobability contours in $\left(\mu_{\eta}, \sigma_{\eta}\right)$ space. For all subsamples and both colors, the lognormal distribution of ellipticities (eq. [28]) can provide as good a fit as the Gaussian distribution (eq. [26]). The best-fitting parameters $\mu_{\eta}$ and $\sigma_{\eta}$, as measured by a $\chi^{2}$ fit to the binned data, are indicated by asterisks in Figure 8 and are listed in Table 1. The best lognormal fit to each subsample yields a median ellipticity that is within $\sim 15 \%$ of that provided by the best Gaussian fit. For all spirals, the median ellipticity is $\epsilon \approx 0.10$ in the $B$ band and $\epsilon \approx 0.16$ in the $K_{s}$ band.

Figure 8 (top) shows the results for the complete sample of spirals. The best fit in the $B$ band, $\mu_{\eta}=-2.36$ and $\sigma_{\eta}=0.96$, is

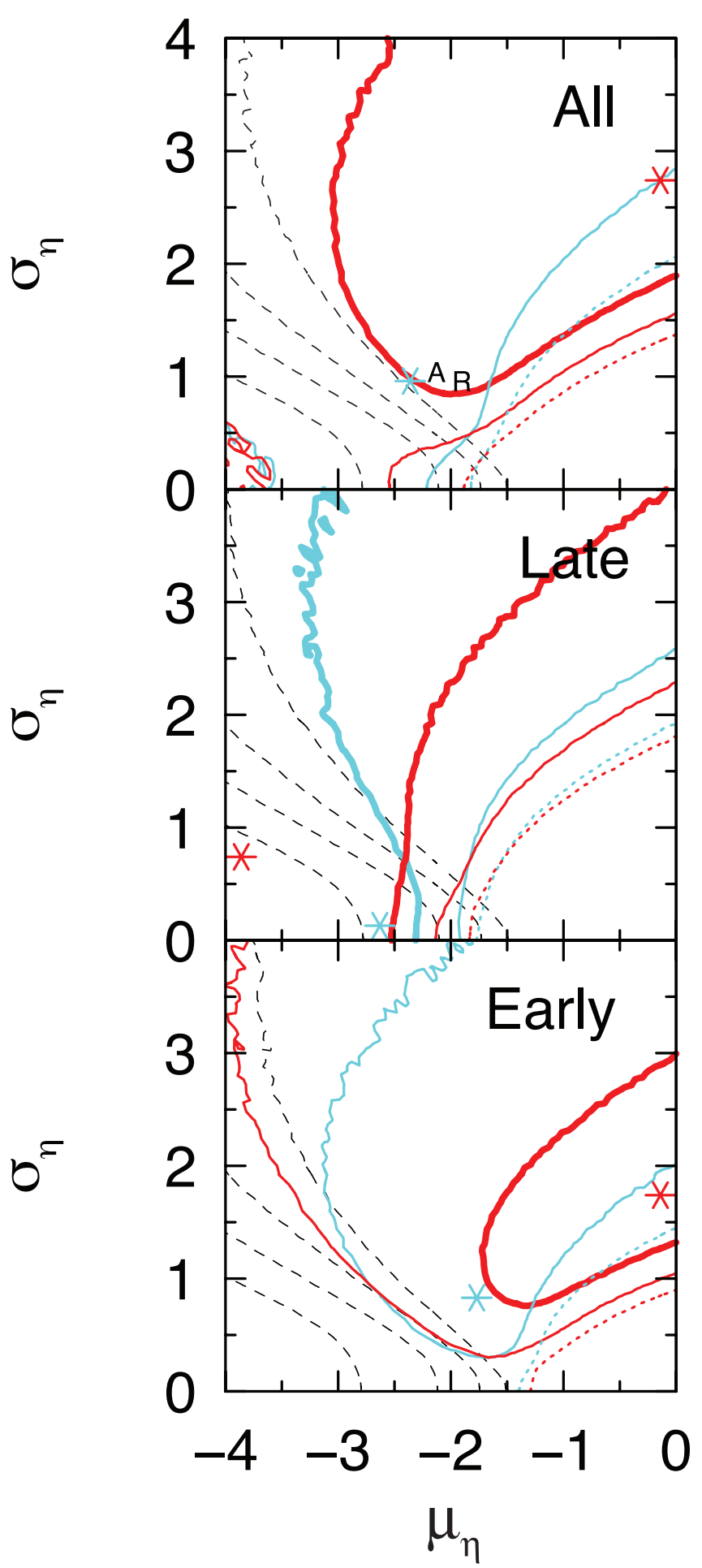

FIG. 8.-Top: Asterisks indicate the best-fitting values of $\mu$ and $\sigma$ to the complete sample of LGA spirals, assuming a lognormal distribution of disk ellipticities. The heavy solid line, light solid line, and dotted line indicate the $P=0.5,0.1$, and 0.01 isoprobability contours. Red indicates shapes deduced from $K_{s}$ data; blue indicates shapes from $B$ data. Dashed lines show the scatter in the Tully-Fisher relation; contours are drawn, starting at the lower left, at $0.25,0.5,0.75$, and 1.0 mag. Middle: Same as top, but for spirals of Hubble type Sc or later. Bottom: Same as top and bottom, but for spirals of type Sbc or earlier. A indicates best fit to data of Andersen et al. (2001); R indicates best fit of Ryden (2004). 
seen to be similar to the best fit found by Ryden (2004) for a sample of exponential galaxies in the Sloan Digital Sky Survey ( $\mu_{\eta}=-2.06$ and $\sigma_{\eta}=0.83$ in the $i$ band). It is also in agreement with the best fit to the sample of Andersen \& Bershady (2002), corrected for their selection criteria $\left(\mu_{\eta}=-2.29\right.$ and $\left.\sigma_{\eta}=1.04\right)$. The best fit in the $K_{s}$ band, $\mu_{\eta}=-0.14$ and $\sigma_{\eta}=2.74$, requires a much broader spread in ellipticities in addition to a greater median ellipticity. However, the values of $\mu_{\eta}$ and $\sigma_{\eta}$ are not very strongly constrained; excellent fits, with $P>0.5$, are produced over a wide range of parameter space with $\mu_{\eta} \gtrsim-1$ and $\sigma_{\eta} \gtrsim 1$.

Figure 8 (middle) shows the results for the late spirals. The best-fitting lognormal distribution in each band implies a small amount of disk ellipticity; the median ellipticity of late spirals is $\epsilon \approx 0.07$ in the $B$ band and $\epsilon \approx 0.02$ in the $K$ band. However, the shapes of late spirals are seen, once again, to be consistent with axisymmetry $\left(\mu_{\eta} \rightarrow \infty, \sigma_{\eta}=0\right)$. The only region of parameter space that is strongly excluded is the lower right corner of the panel, where $\mu_{\eta}$ is large and $\sigma_{\eta}$ is small. Finally, Figure 8 (bottom) shows the results for the early spirals. The best fit in the $B$ band implies a median ellipticity of $\epsilon \approx 0.18$ for the early spirals. The best fit in the $K_{s}$ band implies a still greater degree of disk ellipticity, with a median ellipticity of $\epsilon \approx 0.30$.

\section{IMPLICATIONS AND DISCUSSION}

The 2MASS LGA provides a sample of nearby spiral galaxies with large angular size $\left(R_{3 \sigma}>60^{\prime \prime}\right.$ at $\left.\lambda \sim 2 \mu \mathrm{m}\right)$. In this paper, I have used this relatively small sample of very wellresolved galaxies to estimate the distribution of apparent and intrinsic shapes of spiral galaxies. An intriguing result of this study is the difference in disk ellipticity $\epsilon$ between disk galaxies of early and late Hubble types.

If the entire population of 2MASS LGA spirals is considered, summed over all Hubble types, the distribution of ellipticities in the $B$ band is consistent with earlier photometric studies using larger sample sizes (Lambas et al. 1992; Fasano et al. 1993; Ryden 2004). The $K_{s}$-band ellipticity is greater, but the shape of the $3 \sigma$ isophote in the $K_{s}$ band can be influenced by light from triaxial bulges. The distribution $N_{\epsilon}(\epsilon)$ for shapes in the $B$ band can be modeled as a Gaussian peaking at $\mu_{\epsilon} \approx 0$ and width $\sigma_{\epsilon} \approx 0.16$ or as a lognormal distribution with $\mu_{\eta} \approx-2.4$ and $\sigma_{\eta} \approx 1.0$; both these distributions imply a mean ellipticity $\epsilon \approx 0.1$. When the late spirals, consisting mainly of Hubble type Sc, are examined, they are found to be perfectly consistent with axisymmetry in both the $B$ and the $K_{S}$ band. The early spirals, consisting mainly of types $\mathrm{Sb}$ and $\mathrm{Sbc}$, show a high degree of ellipticity, even in the $B$ band, in which the mean ellipticity is $\epsilon \approx 0.18$.

It has been noted by Franx \& de Zeeuw (1992) that a disk ellipticity of $\epsilon>0.1$ cannot be reconciled with the observed small scatter in the Tully-Fisher relation (Tully \& Fisher 1977) if the disk ellipticity is assumed to trace the potential ellipticity. Suppose that stars and gas are on closed orbits in a logarithmic potential with rotation speed $v_{c}$. If the potential has a small ellipticity $\epsilon_{\phi} \ll 1$ in the orbital plane, then the integrated line profile from the disk will have a width $W=2 v_{c}\left(1-\epsilon_{\phi} \cos 2 \phi\right) \sin \theta$ when viewed from a position angle $(\theta, \phi)$ (Franx \& de Zeeuw 1992). The difference in line width from that produced in a purely circular disk will produce a scatter in the observed TullyFisher relation. If all disks have $\epsilon_{\phi}=0.1$, the expected scatter is $0.3 \mathrm{mag}$, even if the inclination has been determined accurately from kinematic information (Franx \& de Zeeuw 1992). In most studies of the Tully-Fisher relation, the inclination is determined photometrically, from the apparent axis ratio of the disk, assuming (perhaps erroneously) that the disk is axisymmetric. If inclina- tions are determined in this way, the scatter in the Tully-Fisher relation will be even greater.

If galaxies had infinitesimally thin circular disks, the relation between apparent axis ratio $q$ and inclination $\theta$ would be $q=$ $\cos \theta$. If the thin disks actually have ellipticity $\epsilon$ and are viewed at high inclination $\left(\sin ^{2} \theta \gg 2 \epsilon\right)$, the apparent axis ratio will be $q \approx \cos \theta(1-\epsilon \cos 2 \phi)$. Thus, if the inclination is estimated by the relation $\theta_{\text {phot }} \equiv \cos ^{-1} q$, a fractional error $\propto \epsilon \cos 2 \phi$ will be introduced into $\cos \theta_{\text {phot }}$ and an error $\propto \epsilon \cot ^{2} \theta \cos 2 \phi$ will be introduced into $\sin \theta_{\text {phot }}$. Since the observed velocity width $W$ must be divided by $\sin \theta$ to find the rotation speed $v_{c}$, the error produced by using $\sin \theta_{\text {phot }}$ instead of $\sin \theta$ will be negligible only when $\cot ^{2} \theta \ll 1$. Thus, most studies of the Tully-Fisher relation use galaxies of high inclination $\left(\theta_{\text {phot }} \gtrsim 45^{\circ}\right)$. The Tully-Fisher relation for nearly face-on galaxies (see, for instance, Andersen $\&$ Bershady 2003) can only be determined if the inclinations are determined kinematically.

In Figures 7 and 8, the dashed lines indicate the expected amount of scatter in the Tully-Fisher relation if the potential ellipticity is given by either a Gaussian distribution (Fig. 7) or by a lognormal distribution (Fig. 8). Starting at the lower left corner of each panel, the contours are drawn at the levels $0.25,0.5,0.75$, and $1.0 \mathrm{mag}$. The inclinations are assumed to be estimated from the apparent axis ratio. Since the disks are not always infinitesimally thin, the inclination $\theta_{\text {phot }}$ is estimated using the relation

$$
\cos ^{2} \theta_{\text {phot }}=\frac{q^{2}-\mu_{\gamma}^{2}}{1-\mu_{\gamma}^{2}} .
$$

Ultrathin galaxies with $q<\mu_{\gamma}$ are assumed to have $\cos \theta=1$. All galaxies with $\theta_{\text {phot }}<45^{\circ}$ are discarded.

If all spirals are considered together, the best-fitting ellipticity distributions - either Gaussian or lognormal-imply approximately 1 mag of scatter if the potential ellipticity equals the disk ellipticity: this can be seen in the top panels of Figures 7 and 8 . The best-fitting ellipticity for the late spirals, as seen in the middle panels of Figures 7 and 8 , implies only 0.3 mag of scatter. Finally, the best-fitting ellipticity for the early spirals, seen in the bottom panels of Figures 7 and 8, leads to 1.4 mag of scatter in the Tully-Fisher relation. By comparing the predicted scatter from disk ellipticity to the actual scatter in the Tully-Fisher relation for these galaxies, I can place constraints on how much of the scatter can be attributed to the disk ellipticity.

To create a Tully-Fisher plot for the spirals in the 2MASS LGA sample, I took the relevant astrophysical parameters from the HyperLeda database. ${ }^{2}$ As a measure of galaxy luminosity, I used the $I$-band absolute magnitude; multiband studies of the Tully-Fisher relation indicate that the observed scatter is minimized in or near the near-infrared. The $I$-band absolute magnitude is computed from the apparent magnitude $m_{I}$ (corrected for Galactic extinction and internal extinction) and the distance modulus $m-M$. The distance modulus was computed from the radial velocity $v_{\text {Vir }}$, corrected for infall of the Local Group toward Virgo, assuming a Hubble constant $H_{0}=70 \mathrm{~km} \mathrm{~s}^{-1} \mathrm{Mpc}^{-1}$; for galaxies with $v_{\text {Vir }}<500 \mathrm{~km} \mathrm{~s}^{-1}$, the distance modulus was taken from the literature, with preference given to Cepheid distances. As a measure of rotation speed, I used the maximum rotation velocity $v_{m}$ determined from the $21 \mathrm{~cm}$ line of neutral hydrogen. In computing $v_{m}$, the inclination used is the photometric estimate $\theta_{\text {phot }}$, using the axis ratio of the $25 \mathrm{mag} \operatorname{arcsec}^{-2}$ isophote as the apparent axis ratio $q$.

\footnotetext{
${ }^{2}$ See http:// leda.univ-lyon1.fr.
} 


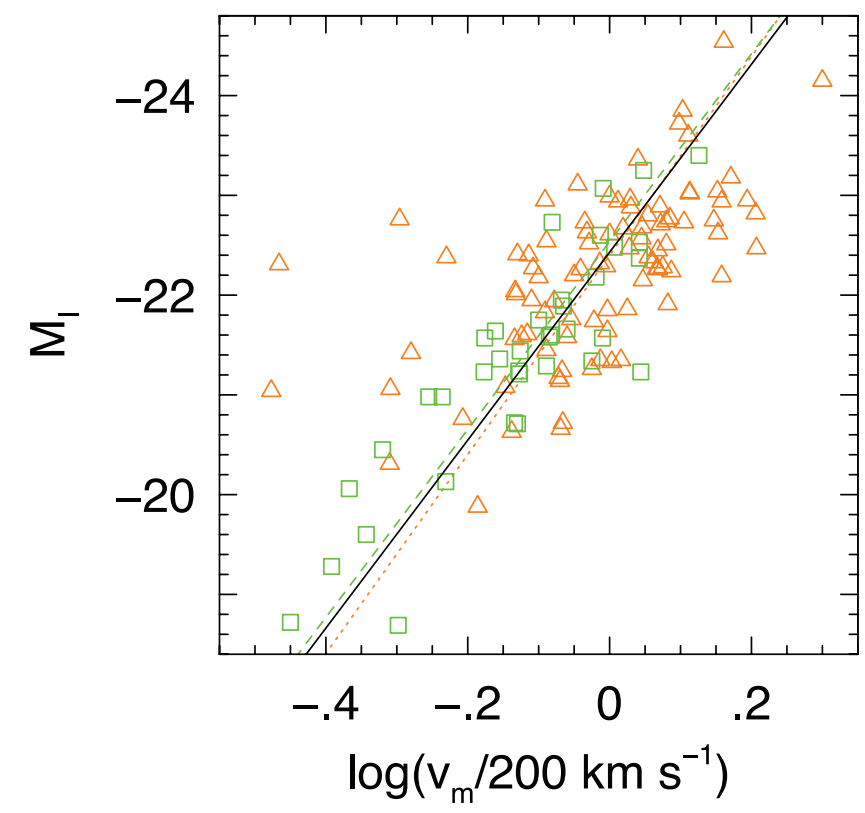

FIG. 9.-Corrected $I$-band absolute magnitude vs. the logarithm of the maximum $21 \mathrm{~cm}$ rotation velocity. Squares indicate late spirals; triangles indicate early spirals. The solid line is the inverse unweighted fit to all the points $(n=128)$, the dashed line is the fit to the late spirals only $(n=37)$, and the dotted line is the fit to the early spirals $(n=91)$.

Figure 9 shows $M_{I}$ as a function of $\log v_{m}$ for the 2MASS LGA spirals with $R_{20}^{0}>60^{\prime \prime}$, assuming axisymmetry; galaxies with $\theta_{\text {phot }}<45^{\circ}$ are omitted, as are galaxies without $I$-band photometry in HyperLeda. A total of $n=128$ galaxies meet all these criteria. The solid line in Figure 9 is the best-fitting TullyFisher relation, found using an unweighted inverse fit (Schechter 1980; Tully \& Pierce 2000; Kannappan et al. 2002). The best Tully-Fisher relation for the sample of $n=128$ galaxies is

$M_{I}($ all $)=(-22.43 \pm 0.09)-(9.42 \pm 0.77) \log \left(\frac{v_{m}}{200 \mathrm{~km} \mathrm{~s}^{-1}}\right)$

with a scatter in $M_{I}$ of $\sigma_{\text {rms }}=0.94$ mag. If only the $n=37$ late spirals are considered, an unweighted inverse fit yields

$M_{I}($ late $)=(-22.54 \pm 0.15)-(9.42 \pm 0.85) \log \left(\frac{v_{m}}{200 \mathrm{~km} \mathrm{~s}^{-1}}\right)$

with a scatter in $M_{I}$ of $\sigma_{\text {rms }}=0.62$ mag. Finally, if only the $n=91$ early spirals are considered, the fit is

$M_{I}($ early $)=(-22.40 \pm 0.12)-(9.97 \pm 1.38) \log \left(\frac{v_{m}}{200 \mathrm{~km} \mathrm{~s}^{-1}}\right)$

with a scatter in $M_{I}$ of $\sigma_{\text {rms }}=1.10 \mathrm{mag}$. The late- and early-type spirals have Tully-Fisher relations with statistically indistinguishable slopes; however, the early spirals have a much greater scatter.

The observed scatter, $\sigma_{\text {rms }}$, is not due entirely to the nonaxisymmetry of disks. In addition to an intrinsic scatter $\sigma_{\text {ell }}$, due to the ellipticity of the disk and the potential, there is also a contribution $\sigma_{\text {err }}$ due to errors in observation and interpretation.
For instance, the absolute magnitude $M_{I}$ is subject to errors in flux measurement, errors in determining distance, and errors in extinction correction. The rotation speed $v_{m}$ is subject to errors in measuring $21 \mathrm{~cm}$ line widths and errors in converting line widths to deduced rotation speeds. Let us suppose that the two sources of scatter add in quadrature: $\sigma_{\mathrm{rms}}^{2}=\sigma_{\text {ell }}^{2}+\sigma_{\mathrm{err}}^{2}$. If $\sigma_{\mathrm{err}}$ is the same for both early and late spirals, then

$$
\begin{aligned}
\sigma_{\text {ell }}(\text { early })^{2}-\sigma_{\text {ell }}(\text { late })^{2} & =\sigma_{\text {rms }}(\text { early })^{2}-\sigma_{\text {rms }}(\text { late })^{2} \\
& =(0.91 \mathrm{mag})^{2}
\end{aligned}
$$

If late spirals are perfectly axisymmetric, which is permitted by the data, then $\sigma_{\text {ell }}$ (late) $=0$ mag and $\sigma_{\text {ell }}$ (early) $=0.91 \mathrm{mag}$. The largest possible values of $\sigma_{\text {ell }}$ come if I naïvely assume that $\sigma_{\text {err }}=0$; in this limiting case, $\sigma_{\text {ell }}($ late $)=0.62 \mathrm{mag}$ and $\sigma_{\text {ell }}($ early $)=1.10 \mathrm{mag}$.

For late spirals, the Tully-Fisher relation thus implies that the intrinsic scatter due to ellipticity lies in the range $\sigma_{\text {ell }}=$ $0-0.62 \mathrm{mag}$. This is consistent with the value $\sigma_{\mathrm{ell}}=0.3 \mathrm{mag}$ derived from the best-fitting distributions of disk ellipticity. For early spirals, the Tully-Fisher relation implies an intrinsic scatter due to ellipticity in the range $\sigma_{\text {ell }}=0.91-1.10 \mathrm{mag}$. This is smaller than the value $\sigma_{\text {ell }}=1.4$ mag derived from the best-fitting distributions of disk ellipticity. However, in the bottom panels of Figures 7 and 8 the band where $\sigma_{\text {ell }}=0.91-1.10$ overlaps the region where $P>0.1$; thus, the ellipticities derived from the apparent shapes and from the Tully-Fisher relation are not discrepant at a high confidence level.

The difference in disk ellipticity between earlier and later spirals, and the resulting difference in Tully-Fisher scatter, helps to explain the apparent discrepancy between the relatively large disk ellipticity $(\epsilon \sim 0.1)$ seen in photometric studies (Binney \& de Vaucouleurs 1981; Grosbøl 1985; Lambas et al. 1992; Fasano et al. 1993; Alam \& Ryden 2002; Ryden 2004) and the relatively small intrinsic scatter $(\sigma \lesssim 0.3 \mathrm{mag})$ seen in some studies of the Tully-Fisher relation. For instance, Verheijen (2001), in his study of spirals in the Ursa Major cluster, found that his DE (distance estimator) subsample of spirals had a Tully-Fisher relation with 0.26 mag of scatter in the $I$ band; the intrinsic scatter had a most likely value of only $0.06 \mathrm{mag}$. This small scatter was partly due to the strict selection criteria imposed; galaxies were excluded if they were obviously interacting, had prominent bars, or had irregular outer isophotes. Galaxies of Hubble types earlier than $\mathrm{Sb}$ or later than Sd were also excluded. The scatter was further minimized by the fact that the resulting subsample of 16 spirals contained 10 galaxies of type Sc, Scd, and Sd (late spirals, in my classification) and only six galaxies of type $\mathrm{Sb}$ and $\mathrm{Sbc}$. If the spirals in the 2MASS LGA are characteristic of all spiral galaxies, then scatter in the Tully-Fisher relation can be reduced by excluding spirals of Hubble type earlier than Sc.

In addition to the practical implications for minimizing scatter in the Tully-Fisher relation, the difference in ellipticity between early and late spirals provides an intriguing clue for the formation and evolution of spiral galaxies. Why should the $\mathrm{Sb}$ and $\mathrm{Sbc}$ galaxies dominating the early spiral sample be more elliptical at $R_{25}$ than the Sc galaxies? One possibility is that the triaxiality of a dark halo's potential affects the Hubble type of the spiral embedded within it. Kormendy \& Kennicutt (2004) estimate that the majority of spirals with Hubble type of $\mathrm{Sb}$ and later contain "pseudobulges" rather than classical bulges. Pseudobulges are formed as gas is transported to small radii by nonaxisymmetric structures such as triaxial halos, spiral structure, elliptical disks, and bars. Simulations of disk galaxies in triaxial 
dark halos (El-Zant \& Hassler 1998; El-Zant 2001) indicate that pseudobulges grow by secular evolution on a timescale of a few Gyr. The Hubble type of the resulting galaxy depends on both the halo core radius and the potential asymmetry, with greater halo nonaxisymmetry leading to larger bulges.

High-resolution $n$-body simulations of virialized dark halos in the mass range $10^{12} M_{\odot} \lesssim M \lesssim 10^{14} M_{\odot}$ indicate that virialized halos are well described as triaxial shapes (Jing \& Suto 2002; Allgood et al. 2006). More massive halos are further from spherical, on average. In a $\Lambda$ CDM simulation, the mean shortto-long axis ratio of a halo with mass $M$ at the present day $(z=0)$ is $\langle\gamma\rangle \approx 0.54\left(M / M_{*}\right)^{-0.05}$, where $M_{*}=1.2 \times 10^{13} M_{\odot}$ is the characteristic nonlinear mass scale (Allgood et al. 2006). The mean intermediate-to-long axis ratio also decreases with increasing mass, going from $\langle\beta\rangle \approx 0.76$ at $M=0.1 M_{*}$ to $\langle\beta\rangle \approx$ 0.71 at $M=M_{*}$. (Dissipation by baryons tends to compress the dark halo into a more nearly spherical shape in its central regions [Kazantzidis et al. 2004], but will not destroy the trend that lower mass halos are rounder on average in the plane of the baryonic disk.) At a given halo mass, there exists a fairly wide spread in $\beta$ around the mean value, with standard deviation $\sigma_{\beta} \sim 0.1$ for $\langle\beta\rangle \sim 0.7$ (Allgood et al. 2006). The nearly axisymmetric halos will give rise to nearly circular disks and smaller pseudobulges, such as those seen in Sc galaxies. The more nonaxisymmetric halos will give rise to elliptical disks and larger pseudobulges, such as those seen in $\mathrm{Sb}$ and $\mathrm{Sbc}$ galaxies. The mean mass of $\mathrm{Sb}$ spirals is greater than that of Sc spirals; however, the spread in masses for a given Hubble type is greater than the difference in the mean (Roberts \& Haynes 1994). This would be expected if the difference between early ( $\mathrm{Sb}$ and $\mathrm{Sbc}$ ) spirals and late $(\mathrm{Sc})$ spirals is not due primarily to differences in halo mass, but rather is due to differences in halo nonaxisymmetry.

This publication makes use of data products from the Two Micron All Sky Survey, which is a joint project of the University of Massachusetts and the Infrared Processing and Analysis Center, California Institute of Technology, funded by the National Aeronautics and Space Administration and the National Science Foundation. It also makes use of the HyperLeda galaxy database. James Pizagno provided useful Tully-Fisher insights.
Abramson, I. S. 1982, Ann. Stat., 10, 1217

Alam, S. M. K., \& Ryden, B. S. 2002, ApJ, 570, 610

Allgood, B., Flores, R. A., Primack, J. R., Kravtsov, A. V., Wechsler, R. H., Faltenbacher, A., \& Bullock, J. S. 2006, MNRAS, in press (astro-ph/0508497)

Andersen, D. R., \& Bershady, M. A. 2002, in ASP Conf. Ser. 275, Disks of Galaxies: Kinematics, Dynamics, and Perturbations, ed. E. Athanassoula \& A. Bosma (San Francisco: ASP), 39 .2003, ApJ, 599, L79

Andersen, D. R., Bershady, M. A., Sparke, L. S., Gallagher, J. S., \& Wilcots, E. M. 2001, ApJ, 551, L131

Beauvais, C., \& Bothun, G. 1999, ApJS, 125, 99

Binney, J. 1978, MNRAS, 183, 779

Binney, J., \& de Vaucouleurs, G. 1981, MNRAS, 194, 679

Bottinelli, L., Gougenheim, L., Paturel, G., \& de Vaucouleurs, G. 1983, A\&A, 118,4

Ciotti, L., \& Bertin, G. 1999, A\&A, 352, 447

Dalcanton, J. J., \& Bernstein, R. A. 2000, AJ, 120, 203

. 2002, AJ, 124, 1328

de Vaucouleurs, G., de Vaucouleurs, A., \& Corwin, H. G. 1976, Second Reference Catalogue of Bright Galaxies (Austin: Univ. Texas)

de Vaucouleurs, G., de Vaucouleurs, A., Corwin, H. G., Jr., Buta, R. J., Paturel, G., \& Fouqué, P. 1991, Third Reference Catalogue of Bright Galaxies (Berlin: Springer)

El-Zant, A. A. 2001, Ap\&SS, 276, 1023

El-Zant, A. A., \& Hassler, B. 1998, NewA, 3, 493

Fasano, G., Amico, P., Bertola, F., Vio, R., \& Zeilinger, W. W. 1993, MNRAS, 262,109

Franx, M., \& de Zeeuw, T. 1992, ApJ, 392, L47

Goad, J. W., \& Roberts, M. S. 1981, ApJ, 250, 79

Grosbøl, P. J. 1985, A\&AS, 60, 261

Guthrie, B. N. G. 1992, A\&AS, 93, 255

Huizinga, J. E., \& van Albada, T. S. 1992, MNRAS, 254, 677

\section{REFERENCES}

Jarrett, T. H., Chester, T., Cutri, R., Schneider, S. E., \& Huchra, J. P. 2003, AJ, 125,525

Jing, Y. P., \& Suto, Y. 2002, ApJ, 574, 538

Kannappan, S. J., Fabricant, D. G., \& Franx, M. 2002, AJ, 123, 2358

Karachentsev, I. D., Karachentseva, V. E., Kudrya, Yu. N., Sharina, M. E., \& Parnovsky, S. L. 1999, Bull. Spec. Astrophys. Obs., 47, 5

Karachentsev, I. D., Karachentseva, V. E., \& Parnovskij, S. L. 1993, Astron. Nachr. 314, 97

Kazantzidis, S., Kravtsov, A. V., Zentner, A. R., Allgood, B., Nagai, D., \& Moore, B. 2004, ApJ, 611, L73

Kormendy, J., \& Kennicutt, R. C. 2004, ARA\&A, 42, 603

Lambas, D. G., Maddox, S. J., \& Loveday, J. 1992, MNRAS, 258, 404

Masters, K. L., Giovanelli, R., \& Haynes, M. P. 2003, AJ, 126, 158

Mitronova, S. N., Karachentsev, I. D., Karachentseva, V. E., Jarrett, T. H., \& Kudrya, Yu. N. 2004, Bull. Spec. Astrophys. Obs., 57, 5

Roberts, M. S., \& Haynes, M. P. 1994, ARA\&A, 32, 115

Ryden, B. S. 1996, ApJ, 461, 146 2004, ApJ, 601, 214

Sandage, A., Freeman, K. C., \& Stokes, N. R. 1970, ApJ, 160, 831

Schechter, P. L. 1980, AJ, 85, 801

Schoenmakers, R. H. M. 1998, in ASP Conf. Ser. 136, Galactic Halos, ed. D. Zaritsky (San Francisco: ASP), 240

Schoenmakers, R. H. M., Franx, M., \& de Zeeuw, P. T. 1997, MNRAS, 292, 349

Silverman, B. W. 1986, Density Estimation for Statistics and Data Analysis (New York: Chapman \& Hall)

Tremblay, B., \& Merritt, D. 1995, AJ, 110, 1039

Tully, R. B., \& Fisher, J. R. 1977, A\&A, 54, 661

Tully, R. B., \& Pierce, M. J. 2000, ApJ, 533, 744

Verheijen, M. A. W. 2001, ApJ, 563, 694

Vincent, R. A., \& Ryden, B. S. 2005, ApJ, 623, 137

Vio, R., Fasano, G., Lazzarin, M., \& Lessi, O. 1994, A\&A, 289, 640 